\title{
Saturated Flow in a Single Fracture: Evaluation of the Reynolds Equation in Measured Aperture Fields
}

\author{
M.J. Nicholl ${ }^{1}$, H. Rajaram², R.J. Glass ${ }^{3}$, and R. Detwiler ${ }^{2}$
}

${ }^{1}$ School of Geology, Oklahoma State University, Stillwater, OK, 74078

${ }^{2}$ Department of Civil, Environmental, and Architectural Engineering, University of Colorado, Boulder CO 80309-0428 Boulder CO 80309-0428
'Flow Visualization and Processes Laboratory, Sandia National Laboratories, Albuquerque, NM EC

Abstract

Fracture transmissivity and detailed aperture fields are measured in analog fractures speclicany $\mathbf{T}$ designed to evaluate the utility of the Reynolds equation. We employ a light transmission technique with well-defined accuracy ( $\sim 1 \%$ error) to measure aperture fields at high spatial resolution $(\sim 0.015 \mathrm{~cm})$. A Hele-Shaw cell is used to confirm our approach by demonstrating agreement between experimental transmissivity, simulated transmissivity on the measured aperture field, and the parallel plate law. In the two rough-walled analog fractures considered, the discrepancy between the experimental and numerical estimates of fracture transmissivity was sufficiently large . $(\sim 22-47 \%)$ to exclude numerical and experimental errors $(<2 \%)$ as a source. We conclude that the three-dimensional character of the flow field is important for fully describing fluid flow in the two rough-walled fractures considered, and that the approach of depth averaging inherent in the formulation of the Reynolds equation is inadequate. We also explore the effects of spatial resolution, aperture measurement technique, and alternative definitions for link transmissivities in the finite-difference formulation, including some that contain corrections for tortuosity perpendicular to the mean fracture plane and Stokes flow. Various formulations for link transmissivity are shown to converge at high resolution $(\sim 1 / 5$ the spatial correlation length) in our smoothly varying fracture. At coarser resolutions, the solution becomes increasingly sensitive to definition of link transmissivity and measurement technique. Aperture measurements that integrate over individual grid blocks were less sensitive to measurement scale and definition of link transmissivity than point sampling techniques. 


\section{DISCLAIMER}

This report was prepared as an account of work sponsored by an agency of the United States Government. Neither the United States Government nor any agency thereof, nor any of their employees, make any warranty, express or implied, or assumes any legal liability or responsibility for the accuracy, completeness, or usefulness of any information, apparatus, product, or process disclosed, or represents that its use would not infringe privately owned rights. Reference herein to any specific commercial product, process, or service by trade name, trademark, manufacturer, or otherwise does not necessarily constitute or imply its endorsement, recommendation, or favoring by the United States Government or any agency thereof. The views and opinions of authors expressed herein do not necessarily state or reflect those of the United States Government or any agency thereof. 


\section{DISCLAIMER}

\section{Portions of this document may be illegible in electronic image products. Images are produced from the best available original document.}




\section{Introduction}

Flow within a rough-walled fracture is three-dimensional, and an exact description should be based on the three-dimensional Navier-Stokes equations. In the limit of very low Reynolds numbers, the three-dimensional Stokes equations may be used. However, the data and computational requirements for solution of the full three-dimensional equations are prohibitive for problems above the microscopic scale. The Reynolds equation, a two-dimensional approximation to the Stokes equations, has been used extensively to evaluate the influence of aperture variability on hydraulic behavior [e.g., Patir and Cheng, 1978; Tsang and Witherspoon, 1981; Brown, 1987, 1989; Gelhar, 1987; Thompson and Brown, 1991;Zimmerman, et al., 1991; Zimmerman and Bodvarsson, 1996; Brown, et al., 1998]. The Reynolds equation was originally developed in the context of lubrication theory [Reynolds, 1886] with the basic assumption that fluid flow behaves locally as in plane Poiseuille flow [e.g., Bird et al., 1960]. In other words, it is assumed that flux components perpendicular to the fracture plane are negligible, and that local transmissivity is given by the "cubic law" for flow between parallel plates.

Application of the Reynolds equation to saturated flow in a single fracture is attractive from a computational standpoint; however, the limitations inherent in the underlying assumptions can potentially lead to inaccurate estimates of effective hydraulic behavior. The validity of the Reynolds equation for saturated fracture flow has been considered in previous theoretical and analytic studies [e.g., Brown et al., 1995; Zimmerman and Bodvarsson, 1996; Ge, 1997]. These studies suggest that in order to maintain a parabolic velocity profile (i.e., negligible hydraulic gradients perpendicular to the mean plane), the mean and variance of the fracture aperture must be significantly smaller than the characteristic length scale of the aperture variation. Also, in order to assure that inertial effects are negligible, fluid velocities must be relatively small. Zimmerman and Bodvarsson [1996] suggest that inertial effects will not be significant for flows at Reynolds number $\left(R_{\text {e }}\right.$ ) smaller than 1 , where aperture is taken as the representative length scale for calculation of $\mathrm{R}_{e}$.

Due to the difficulty in measuring aperture fields, few studies have attempted to test the validity of the Reynolds equation against experimental measurements. Glass et al. [1991] used laser profilometry and light absorption to measure the aperture field of a $30 \times 15 \mathrm{~cm}$ analog fracture. Full field simulations using the Reynolds equation on an aperture field measured by light transmission $(\sim 0.06 \mathrm{~cm}$ resolution) overestimated the experimentally determined transmissivity; the simulated hydraulic aperture was approximately equal to the mean aperture. Simulations on higher resolution data $(0.01 \mathrm{~cm})$ obtained through laser profilometry on a $2.56 \times 2.56 \mathrm{~cm}$ subset of the aperture field yielded transmissivity estimates closer to the measured value. Reimus et al. (1993) measured flow in an $11.6 \times 10.4 \mathrm{~cm}$ natural fracture taken from an outcrop of the Bandelier Tuff. The fracture was taken apart and reassembled between trials; flow measurements showed a factor of two range in the hydraulic aperture between trials. Laser profilometry was used to measure surface topography on both sides of the fracture plane at $0.05 \mathrm{~cm}$ spatial resolution over $a \sim 1 \times 1 \mathrm{~cm}$ region, and at $0.5 \mathrm{~cm}$ resolution on an $11 \times 9.8 \mathrm{~cm}$ region. The two surfaces measured at $0.5 \mathrm{~cm}$ resolution were then matched to model the aperture field; simulations employing the Reynolds equation on this aperture field significantly over predicted experimental estimates of the hydraulic aperture. Mean aperture of the model aperture field was adjusted from an initial value of $0.6181 \mathrm{~mm}$ to $0.0559 \mathrm{~mm}$ in order to obtain a simulated hydraulic aperture within the range of experimental measurements. An independent experimental estimate of the mean aperture was not available, which precludes a rigorous verification of the Reynolds equation. Aperture geometry modeled from the two sets of profilometry data showed different spatial correlation scales; both scales were much smaller than the fracture, hence flow simulations did not show significant channeling.

Hakami and Larsson [1996] measured flow through a branching natural fracture bisecting a $41 \mathrm{~cm}$ long granite core (19 cm diameter). Subsequent to flow experiments, the fracture was filled with fluorescent epoxy and sectioned to obtain aperture data. Details on reconstruction of the aperture 
field from the sectioned data and numerical flow simulations using a 'variable aperture' model are referenced to work in preparation. Numerical simulations predicted a flow rate 2.4 times higher than actually observed. Brown et al. [1998] replicated a $12 \times 7.8 \mathrm{~cm}$ natural rhyolite fracture in transparent epoxy. Transmitted light imaging was used to measure the aperture field at $\sim 0.1 \mathrm{~cm}$ resolution over a $6 \times 6 \mathrm{~cm}$ subset of the fracture plane. Flow simulations on the resulting $64 \times 64$ grid showed large scale channeling similar to that observed during transport of a visible tracer. No direct comparisons were made between simulated and measured flow rates.

Yeo et al. (1998) used an analog system to explore the effects of shear displacement on fracture flow. One surface of a $20 \times 20 \mathrm{~cm}$ natural sandstone fracture was replicated in epoxy. The replica was then used to cast a mating surface. While this methodology might be expected to create two rough surfaces that mate perfectly at zero shear displacement, mismatch between the surfaces resulted in a mean aperture of $\sim 0.6 \mathrm{~mm}$. This observation points to some of the difficulties involved in replicating natural fractures. Spatial structure of the aperture field was measured at 5 mm intervals on silicone rubber casts of the void space between the fracture halves at shear displacements of 0,1 , and $2 \mathrm{~mm}$; fabrication of these casts necessitating disassembling the fracture between flow and aperture measurements. Photomicrographs of the aperture casts clearly show roughness at the sub-millimeter scale, which are not represented in the aperture measurements taken at $5 \mathrm{~mm}$ resolution. Hydraulic aperture was estimated from volumetric flow rate and an average hydraulic gradient that was inferred from measurements made in a limited number of piezometers ( 13 total) located within the fracture plane. Numerical simulations using a finite element approach on the measured aperture field overestimated the experimental hydraulic aperture. The authors concluded that experimental errors were insufficient to account for this observed discrepancy.

In order to fully test the Reynolds equation against experimental data: aperture fields should be measured over the entire fracture at a resolution substantially smaller than the aperture correlation length; fractures should not be disturbed between measurements of aperture field and transmissivity; boundary conditions should be well-defined, closely reflect conditions just inside the fracture plane, and allow for an approximately uniform one-dimensional mean flow; aperture geometry (i.e., mean, variance, spatial correlation) should also be systematically varied. Our objective in this paper is to experimentally test the Reynolds equation in a small number of simple transparent analog fractures; full systematic variation of aperture geometry is outside the scope of this paper In each of our analog fractures, the entire aperture field $(\sim 15 \times 30 \mathrm{~cm})$ is measured at high resolution ( $-0.015 \mathrm{~cm}$, which corresponds to 5 or more points per correlation length) at the time of the hydraulic measurements. To measure aperture fields, we used light absorption techniques [Detwiler et al., in press] that significantly improve on earlier approaches and yield a well defined measurement error for the aperture field. We considered three different test fractures: a Hele-Shaw cell constructed from very smooth glass plates; a rough-walled fracture constructed from two textured glass plates; and a rough-walled fracture constructed from one textured and one smooth glass plate. The Hele-Shaw cell closely approximates a parallel-plate fracture, and therefore serves as a benchmark for our experimental procedure. The rough-walled fractures were designed to represent isotropic, spatially correlated random fields much larger than the correlation length $(\lambda)$ of the field ( $190 \times 400 \lambda)$. In the absence of large-scale channeling, local aperture variation alone controls bulk transmissivity, and thus these aperture fields provide an appropriate challenge for the Reynolds equation. Additionally, for the smooth-rough fracture, aperture measurements completely define the void space geometry and allow a direct assessment of two modified discretizations which attempt to account for deviations from the Reynolds equation; one suggested by $\mathrm{Ge}[1997]$, and another based on Stokes flow in wedge-shaped regions.

Our numerical implementation of the Reynolds equation employed a block-centered finitedifference algorithm on the regular grids generated by the aperture measurements. We considered a number of alternative definitions of the link transmissivity, as well as the two modified discretizations of the Reynolds equation mentioned above. These al temative formulations lead to 
only small differences on the order of $5 \%$ in the estimated effective transmissivity for the roughwalled fractures considered. Including error in the measured aperture field, as well as variation in the formulation for link transmissivity, computational estimates of the effective transmissivity overlapped experimental measurements for the Hele-Shaw cell, and over predicted effective transmissivity by 22 to $34 \%$ for the rough-rough cell, and 33 to $47 \%$ for the smooth-rough cell. The discrepancies between simulation and experiment for the rough-walled cells are far in excess of the error in measured transmissivity $(-1.3 \%$ to $+0.5 \%)$. We also considered the influence of spatial resolution in the aperture field on our simulation results by both coarsening the measured aperture fields, and by additional simulations performed on re-measured higher resolution aperture data. Results confirmed that our measurements and simulations on the rough-walled fields were at sufficient resolution ( $1 / 5$ the aperture spatial correlation length) to yield predictions within $2 \%$ of the fully converged solution. Therefore, the discrepancy between theoretical predictions and experimental results points to the importance of the full three-dimensional aspects of flow within these simple, spatially correlated, aperture fields. We also observed that discretization coarser than the aperture spatial correlation length significantly impacts simulation results; both as a function of the choice of link transmissivity and the aperture field measurement method (area averaging or point). The choice of link transmissivity has a smaller influence on simulation results when data is obtained using measurement methods that integrate over individual grid blocks as opposed to point data. In studies of the small scale physics of flow and transport in fractures where the flow field is of critical importance, our results suggest that caution should be exercised in the use of twodimensional approaches such as the Reynolds equation that inadequately represent the true threedimensional nature of the flow field. Furthermore, our results suggest that both the scale of aperture measurement, and the measurement technique must be carefully considered during experimental design.

The paper is organized as follows: The experimental techniques are described in detail in Section 2. Aperture measurements and statistical properties of each aperture field considered are provided in Section 2.1. The flow experiments are discussed in Section 2.2, along with theoretical estimates based on the stochastic theory of flow in random porous media. Numerical implementation of the Reynolds equation is described in Section 3. Alternative formulations of the link transmissivity are introduced in Section 3.1, and the sensitivity of those formulations to local aperture variability is explored in Section 3.2. In Section 3.3 we compare computational estimates of the effective transmissivity to the experimental measurements. Section 4 considers the influence of resolution in the aperture measurement on simulation results. The paper concludes in Section 5 with a summary, a discussion of the important results, and potential avenues for further research.

\section{Physical measurements}

A critical design feature of our experiments is that the fracture is not disturbed between measurements of aperture field and transmissivity. We accomplish this by employing transmitted light imaging techniques reported by Detwiler et al. [in press] to measure the aperture field at the time of the hydraulic tests. Their methodology not only improves on earlier approaches [e.g., Glass et al., 1991; Glass, 1993; Nicholl and Glass, 1994; Persoff and Pruess, 1995; Glass and Nicholl, 1995; Brown, et al., 1998], but also provides a means for quantifying and minimizing measurement error. Each of our three test fractures was assembled within a test cell that allows us to transmit light through the entire fracture plane, impose boundary conditions on the fracture edges, and apply normal pressure to the fracture plane (see Figure 1). Light from a feedback stabilized source was transmitted through the fracture and measured by an electronically cooled high resolution CCD camera (2045 x 2033 pixels, 4096 gray levels). The test cell was mounted on a test stand that holds it in rigid alignment with the camera and light source. Full details on this measurement system are given in Detwiler et al. [in press]. 
We measured the transmissivity and aperture fields of three transparent analog fractures: a HeleShaw cell constructed from very smooth glass plates; a rough-walled fracture constructed from two textured glass plates; and a rough-walled fracture constructed from one textured and one smooth glass plate. The Hele-Shaw cell was fabricated from two pieces of $1 / 2$ " $(1.27 \mathrm{~cm})$ thick plate glass polished flat to a maximum deviation of $0.0001^{\prime \prime}\left(2.54 \times 10^{-4} \mathrm{~cm}\right)$. The glass plates $(19 \times 30.4 \mathrm{~cm})$ were clamped directly in the test cell without application of a normal pressure and separated by plastic shims (nominal thickness of $0.0178 \mathrm{~cm}$ ) that also served as no flow boundaries on the long edges (actual flow width of $15.23 \mathrm{~cm}$ ). Geometry of this system was designed to closely conform with the assumptions underlying the Reynolds equation (i.e., local transmissivity is fully explained by the cubic law), and therefore, provide an independent check on our methodology.

Rough-walled analog fractures were fabricated using textured glass. Fracture dimensions ( $14.8 \mathrm{x}$ $30.2 \mathrm{~cm}$ ) were chosen to minimize boundary effects and create an isotropic, spatially correlated random aperture field much larger than the correlation length $(\lambda)$. In the absence of the large-scale channeling that would result from system-scale heterogeneity, local aperture variation controls bulk transmissivity, and thus these aperture fields provide a fitting test for the Reynolds equation. A "rough-rough" fracture was fabricated by holding two pieces of the textured glass in face-to-face contact. A normal pressure (20 psi) was applied to the fracture plane in order to minimize long wavelength features in the aperture field, and was held constant throughout the entire experiment. We have previously used equivalent fractures to study: gravity-driven instability [Nicholl et al., 1993a,b; Nicholl et al., 1994]; modified invasion percolation in fractures [Glass, 1993; Glass, et al., 1997; Glass, et al., 1998]; unsaturated flow and transport [Nicholl and Glass, 1994]; and entrapped phase dissolution [Glass and Nicholl, 1995]. We also consider a "smooth-rough" fracture fabricated by holding a piece of the same textured glass against a piece of smooth glass under 20 psi of normal pressure. In this smooth-rough configuration, aperture measurements fully establish aperture geometry, permitting evaluation of modified formulations which incorporate deviations from the Reynolds equation.

\section{1 measurement and description of aperture fields}

We measured aperture fields using light transmission theory first proposed by Glass et al. [1991]. The measurement protocol outlined by Detwiler et al. [in press] was employed to minimize and quantify measurement errors that result from a combination of image noise, nonlinear dye absorbance, and refraction within the fracture. In this protocol, we first measure the aperture field (normalized to its mean) and evaluate the total root mean squared (RMS) error; mean aperture and associated error are measured independently. Errors in both the mean and the normalized aperture field are combined using a relation derived through a perturbation approach. Implementing this protocol, each fracture was filled with a series of solutions $(0,1 / 32,1 / 16,1 / 8$, and $1 / 4 \mathrm{~g} / \mathrm{l}$ of FD\&C Blue Dye \#1); a set of 80 images was collected at each concentration. Each image was then adjusted for small temporal fluctuations in the light source using measured intensities at a stepped neutral density wedge located adjacent to the test cell (see Figure 1). After checking each image for spatial shifts (none detected), we then averaged each set of intensity adjusted images to produce a single, reduced noise image for each concentration. We then checked and corrected for spatial shifts between each average image (maximum shift of 0.16 pixels) and for parallax. This series of average images was used to determine the single dye concentration that minimizes total RMS error by balancing errors in precision (image noise) and accuracy (dye absorbance). For each of the fractures described here, we found the $1 / 16 \mathrm{~g} / \mathrm{l}$ concentration to yield the smallest total error. We chose not to match the index of refraction of the fluid and fracture walls, increasing RMS error in the rough-rough fracture by $0.9 \%$ relative to its mean aperture. Combining precision and accuracy based errors yields total RMS error relative to the respective mean apertures of the normalized aperture fields ( $1.5 \%$ for the rough-rough, $1.4 \%$ for the smooth-rough, and $0.8 \%$ for the HeleShaw cell). Injection of a known volume of fluid into the fractures allowed measurement of the mean aperture to within $\pm 0.7 \%$ for all three fractures. Total combined error of the dimensional 
aperture field (equation 12, Detwiler et al. [in press]) is also less than $\pm 1 \%$ of the mean aperture for all three fractures. Spatial resolutions of the aperture field measurements were $0.0149 \mathrm{~cm}$ for the rough-walled fractures, and 0.0151 for the Hele-Shaw cell.

Aperture distributions for the three fractures are shown in Figure 2. The Hele-Shaw cell shows a narrow distribution about the mean aperture, with values ranging from about $0.018-0.021 \mathrm{~cm}$. The aperture variance is only $8.12 \times 10^{-07} \mathrm{~cm}^{2}$, giving a coefficient of variation of $2.387 \times 10^{-02}$. The smooth-rough fracture exhibits a negatively skewed distribution with a strong peak and abrupt truncation on the upper end. The rough-rough fracture also has a negatively skewed distribution, which is much wider than that of the smooth-rough fracture. The mean aperture in the rough-rough fracture is a little less than twice that of the smooth-rough fracture (Table 1), suggesting that some degree of interlocking occurred between the surfaces. The coefficient of variation in the smoothrough aperture field is about $30 \%$ higher than in the rough-rough fracture.

Representative segments of the rough-walled aperture fields are shown in Figure 3; note that the smooth-rough aperture field clearly shows the pattern of the textured glass used in both fractures. It may be noted from Figure 2 that no zero aperture values occur in either of the rough-walled fractures. This is because the light transmission technique measures average aperture within a pixel. As a result, zero aperture values will not be recorded unless contact areas are at least pixelsized. Detailed analysis of small aperture regions in the smooth-rough aperture field (see Figure 3), and optical inspection of the textured glass itself show that the raised bumps on the glass have a definite rounded crest. We expect these rounded crests to result in contact patches or points of negligible area that are scattered about the aperture field at separation distances much greater than the correlation length.

Visual examination of the aperture fields revealed large scale features in all three fractures, albeit to a minor degree. This was surprising in the Hele-Shaw cell, considering the care taken in fabrication. Aperture variability was characterized by computing semi-variograms for all three fractures along the primary and diagonal axes. Variograms for the Hele-Shaw cell (Figure 4) show anisotropy, which results from large scale features. Data indicates a slight narrowing of the aperture field near the lateral boundaries and a slight trend along the principal axis. We surmise that clamping pressure imparted a slight bowing across the short axis of the cell and led to unequal compression of the shim material along the long axis. The magnitude of these variations are sufficiently small (Table 1) that the Hele-Shaw cell is expected to behave as a parallel plate fracture.

Variograms for both rough-walled fractures show that the spatial structure of the aperture fields are dominated by strong short range correlation at separation distances on the order of $-0.08 \mathrm{~cm}$ (Figure 4). Short-range variograms are essentially identical for the two systems, showing a marginally longer correlation length for the short axis than the long axis. The repetitive nature of the patterned glass (see Figure 3$)$ induces a negative correlation at $(-0.08-0.18 \mathrm{~cm})$; i.e., regions of correlated small aperture are adjacent to regions of correlated large aperture. The manufacturing process (hot rolling) overlays a mild large scale structure ( $\rightarrow 3$ cycles over the fracture width) that can be seen by eye in full scale images of the smooth-rough aperture field. This structure is largely broken up in the rough-rough fracture. The small scale spatial correlation seen in Figure 4 overwhelms the large scale pattern so that it does not appear in the variograms. Based on tracer tests in fractures equivalent to the rough-rough analog [Nicholl and Glass, 1994] and observations during aperture measurement, we infer that the large scale structure does not induce any channeling at the system scale. Therefore, statistically similar subsets of the measured aperture fields should behave in a manner similar to the entire fields. 


\subsection{Fracture transmissivity}

At small Reynolds number $\left(R_{e}\right)$, the relationship between differential head $(\Delta h)$ and volumetric flow rate (Q) of an incompressible fluid through a horizontal fracture of width (W) and length (L) is given by Darcy's Law [e.g., Bear, 1979]:

$$
Q=T W \frac{\Delta h}{L}
$$

where $T$ represents fracture transmissivity. For flow through an aperture (b) separating parallel plates, T is given by the "cubic law" (e:g., Bear, 1993):

$$
T=\frac{b^{3} g}{12 v}
$$

where $g$ is the acceleration due to gravity, and $v$ is kinematic viscosity. The expression (2) has been used previously [e.g., Hakami, 1989; Piggot and Elsworth, 1993; Reimus et al., 1993; Waite, et al., 1998] to calculate an effective hydraulic aperture $\left(b_{h}\right)$ from experimental estimates of fracture transmissivity. As $b_{h}{ }^{3}$ is independent of fluid properties, we chose this measure to compare experimental and numerical results.

In each of our fractures, the long edges were held as no-flow boundaries, and steady state flow of deionized water was induced between full width manifolds covering the short edges (see Figure 1). We first increased, and then decreased $\Delta h$ through a series of steps by altering the heights of a constant head reservoir on the inflow side and a stabilized drip point on the outflow. Volumetric flow rate was measured gravimetrically at each step $( \pm 0.0002 \mathrm{~g})$ and differential head was obtained from manometers attached to the inlet and outlet manifolds $( \pm 0.05 \mathrm{~cm})$. Fluid temperature measured in the inlet reservoir $\left( \pm 0.5^{\circ} \mathrm{F}\right)$ was used to calculate kinematic viscosity of pure water from standard tables. Precision errors are expected to be random, and hence may be calculated from the measured data. Accuracy of the thermometers leads to a potential error in viscosity of \pm $0.5 \%$. Post-experimental evaluation of the test cell leads us to conclude that the manometer and manifold design caused our experimental measurements to slightly underestimate differential head across the fracture. Each manifold (inflow and outflow) had a manometer port on one end, and a fluid inlet/outlet port on the opposite end (Figure 1). Distribution of fluid along the manifold requires an expenditure of energy that is proportional to flow rate. Therefore, the manometers slightly underestimated average head within the inlet manifold and overestimated average head in the outlet manifold. By assuming that the manifolds acted like leaky capillary tubes, we were able to estimate potential bias in the differential head measurements at $+0.82 \%$ for the Hele-Shaw cell, $+0.12 \%$ for the smooth-rough fracture, and $+0.83 \%$ for the rough-rough fracture..

In order to calculate $b_{h}{ }^{3}$ from our experimental data, we combine (1) and (2) to yield:

$$
Q \frac{12 \nu}{W g}=b_{h}^{3} \frac{\Delta h}{L}
$$

Figure 5 shows experimental data with the left-hand side of (3) plotted as a function of hydraulic gradient $(\Delta \mathrm{h} / \mathrm{L})$. The straight line fits to the data imply Darcy flow. The slope of best-fit lines passing through the origin are used to estimate $b_{h}{ }^{3}$. The regression statistics (Table 2) show exceptionally high $\mathrm{R}^{2}$ for all three fractures and narrow bands for the $68 \%$ confidence intervals $( \pm 1$ standard deviation). Error bounds are placed on $b_{h}{ }^{3}$ by adding accuracy errors (viscosity, 
differential head) to the $68 \%$ confidence intervals, which serve as an estimate of precision error (Table 2). Mean aperture was used as the characteristic length scale to calculate $\mathrm{R}_{c}$ for the experimental data. With the exception of one experimental trial in the rough-rough fracture, volumetric flow varied linearly with hydraulic gradient over the ranges of $R_{e}$ that were considered $e$ (Table 2). One experiment in the rough-rough fracture that was run at relatively high Reynolds number $\left(R_{c}=6.8\right)$ plots slightly below the linear fit through the remaining data, suggesting nonDarcy flow (inertial effects); data from this trial was not included in the regression used to estimate $b_{h}{ }^{3}$. Employing the cube of mean aperture $\left(\langle b\rangle^{3}\right)$ and associated error to estimate $b_{h}{ }^{3}$ gives results that fall within the error bounds for the Hele-Shaw cell, but leads to errors of $+40-49 \%$ in the rough-rough fracture and $+66-76 \%$ in the smooth-rough fracture.

Experimental measurements of transmissivity can be compared to predictions based on stochastic theory. Zimmerman and Bodvarsson [1996] discuss the following predictor of transmissivity in a two-dimensional, isotropic random medium:

$$
T_{\text {eff }}=\langle T\rangle\left(1-\frac{\sigma_{T}^{2}}{2\langle T\rangle^{2}}\right)
$$

Expression (4), which predicts that effective transmissivity $\left(T_{\text {dff }}\right)$ will decrease as the variance of local transmissivity increases was obtained by Landau and Lifshitz [1960] and is valid for smooth aperture variations, regardless of the actual aperture distribution. The above expression can also be viewed as a perturbation approximation to the geometric mean of the transmissivity, which is the appropriate effective transmissivity for a two-dimensional isotropic medium with lognormal transmissivity distribution, according to the theories of Bakr et al. [1978] and Dagan [1979]. Substitution of (2) into (4) allows prediction of $b_{h}{ }^{3}$ from the aperture statistics. While the results overestimate the experimental $b_{h}{ }^{3}$ values for the rough-rough and smooth-rough fractures by 32$35 \%$ and $56-58 \%$ respectively, they do provide a first-order improvement over estimates based solely on mean aperture.

\section{Numerical Solution of the Reynolds equation}

The Reynolds equation is based on the assumption that the local behavior of fluid flow between two rough surfaces is similar to flow between two parallel plates. It is assumed that hydraulic gradients and velocity components perpendicular to the fracture plane can be neglected for flow at small Reynolds number in fractures that exhibit gradual aperture variation. Zimmerman and Bodvarsson [1996] give $R_{c}=1$ as a conservative upper bound on the applicability of the Reynolds equation. Under such conditions, local velocity profiles across the aperture are expected to be parabolic, as in plane Poiseuille flow. Integration of the velocity profile across the aperture leads to a linear relationship between the fluid flux and the hydraulic gradient in the fracture plane. By analogy to two-dimensional flow in porous media, this leads to the definition of transmissivity (T) given by (2).

The Reynolds equation can then be written as:

$$
\frac{\partial}{\partial x}\left\lceil\frac{b(x, y)^{3} g}{12 v} \frac{\partial h}{\partial x}\right]+\frac{\partial}{\partial y}\left[\frac{b(x, y)^{3} g}{12 v} \frac{\partial h}{\partial y}\right]=0
$$

where $\mathrm{h}$ is the piezometric head (sum of pressure and elevation head), $\mathrm{x}$ and $\mathrm{y}$ are Cartesian coordinates in the plane of the fracture. Variability in the aperture field is incorporated in (5), which is similar to two-dimensional flow in a heterogeneous porous medium [Tsang and Tsang, 1989]. The head field $h(x, y)$ may be computed through a numerical solution of (5); we employed a 
standard block-centered finite-difference approach [e.g., Bear, 1979]. The finite-difference discretization of $(5)$ is obtained by writing the flux balance for the grid block centered at $x=i \Delta x, y$ $=\mathrm{j} \Delta \mathrm{y}$ as follows:

$$
\begin{aligned}
& \Delta y \frac{b_{i+1 / 2, j}^{3} g}{12 v}\left[\frac{h_{i+1, j}-h_{i, j}}{\Delta x}\right]-\Delta y \frac{b_{i-1 / 2, j}^{3} g}{12 v}\left[\frac{h_{i, j}-h_{i+1, j}}{\Delta x}\right]+ \\
& \Delta x \frac{b_{i, j+1 / 2}^{3} g}{12 v}\left[\frac{h_{i, j+1}-h_{i, j}}{\Delta y}\right]-\Delta x \frac{b_{i, j-1 / 2}^{3} g}{12 v}\left[\frac{h_{i, j}-h_{i, j-1}}{\Delta y}\right]=0
\end{aligned}
$$

where $\Delta \mathrm{x}$ and $\Delta \mathrm{y}$ are the grid block dimensions in the $\mathrm{x}$ and $\mathrm{y}$ directions, respectively. The quantities such as $b^{3}+1 / 2, j$ and $b^{3}{ }_{i-1 / 2, j}$ in (6) are the "link transmissivities", which are typically approximated as some average of the block transmissivities. We next consider the influence of several alternative expressions for the link transmissivity, noting that in a smoothly varying aperture field, all such expressions will converge to $b^{3}$.

\subsection{Definition of link transmissivity}

The two-dimensional approximation resulting from application of the Reynolds equation is identical to two-dimensional flow in a porous medium, with the transmissivity interpreted as in (2). Thus, one approach to defining link transmissivities is to follow established approximations used in the porous media literature. Bear [1979] suggests the arithmetic and harmonic averages of the block transmissivities as alternatives for approximating link transmissivity:

"arithmeticaverage"

$$
b_{i+1 / 2, j}^{3}=\frac{b_{i, j}^{3}+b_{i+1, j}^{3}}{2}
$$

"harmonic average"

$$
b_{i+1 / 2, j}^{3}=\frac{2 b_{i, j}^{3} b_{i+1, j}^{3}}{b_{i, j}^{3}+b_{i+1, j}^{3}}
$$

Bear [1979] notes that the harmonic average (7b) is a consistent definition of the equivalent transmissivity for one-dimensional flow between adjacent grid blocks. Cordes and Kinzelbach [1996] show that mixed finite element formulations on triangular elements obtained by dividing rectangular elements, give fluxes across the rectangle boundaries identical to those obtained from block-centered finite-difference formulations using (7b). Standard finite element formulations, however, correspond to (7a).

Brown [1987, 1989] and Brown et al. [1998] assumed that the arithmetic mean of the apertures in adjacent grid blocks would provide a representative aperture for computing the link transmissivity (Personal Communication, S.R. Brown, 1998), leading to:

"midpoint"

$$
b_{i+1 / 2, j}^{3}=\left[\frac{b_{i, j}+b_{i+1, j}}{2}\right]^{3}
$$

For two-dimensional flow in a porous medium with lognormally distributed transmissivity, stochastic theories [e.g., Dagan, 1989; Gelhar, 1993; Zimmerman and Bodvarsson, 1996] predict that effective transmissivity will be given by the geometric average: 
While the approximations (7a-d) are justifiable as some type of average transmissivity, they do not consider aperture geometry. An alternative approach to defining link transmissivities involves analytical solutions to laminar flow in approximate fracture geometries. If fracture geometry between adjacent grid blocks is approximated as a wedge (Figure 6), then solutions to the Reynolds and Stokes equations can be invoked to develop expressions for the link transmissivity. Solution of the Reynolds equation for one-dimensional flow in a wedge (i.e., Figure 6a) leads to:

"Reynolds wedge" $\quad b_{i+1 / 2, j}^{3}=\frac{2 b_{i, j}^{2} b_{i+1, j}^{2}}{b_{i, j}+b_{i+1, j}}$

It should be noted that (7e) is solely dependent on the individual apertures, and does not consider the wedge angle; however, the underlying assumption of $1-D$ flow will become increasingly tenuous as the wedge angle becomes large.

In our experimental approach, it is the fracture aperture that is measured, and not the topography of the individual fracture surfaces; however, as noted previously, the aperture measurements are sufficient to specify aperture geometry for the smooth-rough fracture (Figure 6b). We exploit that geometry (i.e., wedge angle) to develop two other expressions for link transmissivity. Twodimensional Stokes flow in a wedge-shaped region, known as Hamel's problem [Langlois, 1964] is amenable to exact solution. Unlike in the Reynolds equation, pressure variation across the aperture is not neglected. Instead, flow is assumed to be "radial", with the two walls of the wedge as bounding radii on which no-slip boundary conditions are imposed (Figure 6c). Using the solution to Hamel's problem, a linear relationship between flow rate and gradient of the depthaveraged pressure can be obtained, leading to:

"Stokes wedge"

$$
b_{i+1 / 2, j}^{3}=\frac{2 b_{i, j}^{2} b_{i+1, j}^{2}}{b_{i, j}+b_{i+1, j}} \frac{3(\tan \theta-\theta)}{\tan ^{3} \theta}
$$

where $\theta$ represents the wedge angle as illustrated in Figure 6b. Note that $(7 f)$ is independent of the direction of flow (converging or diverging), which is consistent with creeping flow at low Reynolds number [e.g., Longwell, 1960]. At moderate values of the Reynolds number, flow separation in the divergent wedge would be expected to invalidate this approximation.

A recent modification to the Reynolds equation [Ge, 1997] ignores pressure gradients perpendicular to the center-line of the wedge, rather than those in the vertical direction. In effect, the Reynolds equation is applied in a coordinate system aligned with the center-line of the wedge. Two modifications to the Reynolds equation result, one accounting for the change in path length and the other for aperture measured perpendicular to the center-line; these modifications lead to:

"Modified Reynolds wedge"

$$
b_{i+1 / 2, j}^{3}=\frac{2 b_{i, j}^{2} b_{i+1, j}^{2}}{b_{i, j}+b_{i+1, j}}\left(\frac{2 \cos \theta}{\cos \frac{\theta}{2}(1+\cos \theta)}\right)^{3}
$$

Aperture measurements do not fully define geometry of the rough-rough fracture. We do however know that it varies in both directions from the mean plane; therefore, we assume that recasting ( $7 f$ ) in terms of a symmetric wedge (Figure $6 \mathrm{~d}$ ) will provide a better approximation of the actual geometry than the asymmetric wedge used as a basis for both (7f) and (7g): 
"Symmetric wedge"

$$
b_{i+1 / 2, j}^{3}=\frac{2 b_{i, j}^{2} b_{i+1, j}^{2}}{b_{i, j}+b_{i+1, j}} \frac{3(1-\theta \cot \theta)}{4 \tan ^{2}(\theta / 2)}
$$

While the wedge angle does not influence (7e), (7f-h) include corrections to (7e) that will act to decrease link transmissivity as the wedge angle increases. Figure 7 shows the ratio of link transmissivity predicted by expressions (7f-h) relative to (7e) as a function of wedge angle. For wedge angles $<10^{\circ}$, the corrections imposed by $(7 \mathrm{f}-\mathrm{h})$ are small $(<2 \%)$, however, as wedge angle approaches $90^{\circ},(7 \mathrm{f}-\mathrm{h})$ predict that link transmissivity will approach zero. As noted previously, the link transmissivities (7f-h) were obtained after depth-averaging the pressure distribution within the wedge. As the wedge angle increases, the pressure variation across the depth would be so large that a description in terms of the averaged pressure is perhaps not very useful. Furthermore, complex three-dimensional flow patterns can be expected at the boundaries between two adjacent "wedges". Such interactions between adjacent wedges are not accounted for in the simple analytical solutions that form the basis for (7f-h). Therefore, although it is reassuring that the link transmissivities (7f-h) reveal significant deviations from the Reynolds equation for large wedge angles, they should not be viewed as a substitute for a rigorous three-dimensional analysis.

\subsection{Sensitivity of link transmissivity to local aperture variability}

Before proceeding to numerical simulations, we consider differences between formulations for link transmissivity given by (7a-g) as a function of the change in aperture between adjacent grid points, expressed as a ratio $\left(r=b_{i+1, j} \div b_{i, j}\right)$. Table 3 summarizes the limiting behavior of the link transmissivities (7a-g) as $r \rightarrow \infty$. Predictions based on (7a) and (7c) grow with $r^{3}$, (7d) grows with $\mathrm{r}^{3 / 2}$, and (7e) grows linearly. Predictions based on (7b) and (7h) approach a limit as $\mathrm{r} \rightarrow \infty$, while (7f) and (7g) go to zero. The link transmissivities predicted by (7b) are compared to (7e-h) in Figure 8, for a value of $\Delta x / b_{i, j}=1$. The link transmissivity (7f) increases with $r$, up to a value of $r$ $=1.8$ and then decreases, approaching zero as $\mathrm{r} \rightarrow \infty$. The values of $\mathrm{r}=1.8$ corresponds to $\mathrm{a}$ wedge angle of about $38^{\circ}$ for $\Delta x / b_{i, j}=1$. This value of wedge angle is quite large and is perhaps in a regime where three-dimensional analysis is warranted. The link transmissivity $(7 \mathrm{~g})$ behaves very much like (7f), although it is slightly larger, except at wedge angles larger than $75^{\circ}$. The link transmissivity $(7 \mathrm{~h})$ grows with $\mathrm{r}$ and approaches a constant value given by $\left(3 \pi \mathrm{b}_{\mathrm{i}, j} / 2 \Delta \mathrm{x}\right)$ at large values of $r$. For values of $b_{i, j} / \Delta x<0.42$, the limiting value predicted by $(7 \mathrm{~h})$ is smaller than that predicted by $(7 b)$. It is interesting to note that $(7 f-h)$ predict a link transmissivity smaller than the value predicted by (7b) under certain conditions. The harmonic average is widely regarded as the absolute lower bound on the effective transmissivity of a random conductive medium, provided that flux at all locations is linearly dependent on the local hydraulic gradient [e.g., Dagan, 1979]. The expressions (7f-h) can however become smaller than (7b), since they are based on the Stokes equations and hence include the effects of flow components perpendicular to the fracture plane.

\subsection{Flow simulations on the measured aperture fields}

The expressions (7a-h) were all used in the two-dimensional computations of fluid flow within the measured aperture fields. The simulated domain consisted of a region with dimensions of $27.9 x$ $12.9 \mathrm{~cm}$ inside the test cells ( $1876 \times 866$ grid points). The peripheral regions of the measured aperture field were trimmed from the domain to eliminate linear artifacts resulting from the internal edges of the clear gaskets used for the confinement cell (see Figure 1). Because the aperture field is statistically homogeneous with a spatial correlation scale of only about $0.08 \mathrm{~cm}$, this trimming should not influence the effective transmissivity estimates. The simulated domain extends over roughly $350 \times 160$ correlation scales. No flux boundaries were imposed along the lateral edges of the domain. Constant head boundaries were imposed in cells just outside the longitudinal 
boundaries; aperture of the constant head cells was set to the fracture mean. The sparse system of linear equations resulting from (5) was solved using a preconditioned conjugate gradient algorithm. The Modified Implicit Factorization approach [Larabi and de Smedt, 1994] was used for preconditioning. A linear variation of the head between the inflow and outflow boundary was specified as an initial trial solution; flux balance error was defined as the absolute value of (cumulative inflow-cumulative outflow)/(cumulative inflow). The iterative solver was required to reduce the RMS flux balance error to $10^{-9}$ times that associated with the initial trial solution, while maintaining a flux balance error of less than $0.1 \%$. In most cases, a flux balance error of less than $0.001 \%$ resulted.

Flow rate $(\mathrm{Q})$ through each fracture was computed from the numerical solution and used to calculate effective transmissivity based on (3). Working with analog fractures equivalent to the ones considered here, Detwiler et al. [in press] showed that changes in aperture distribution on the order of $5 \%$ RMS had a relatively small effect $(-3.5 \%)$ on predictions of $b_{h}{ }^{3}$. As our RMS errors are much smaller $(\sim 1 \%)$ we assumed that errors in the aperture distribution would have a negligible impact on prediction of $b_{h}^{3}$. Therefore, we employed the error bounds on the experimentally determined aperture mean to set upper and lower bounds on our simulation estimates for $b_{h}{ }^{3}$; results of our simulations on the measured aperture field are summarized in Table 4 for each of the different link transmissivity expressions. For the Hele-Shaw cell, the variation between the estimates obtained using the largest and smallest link transmissivity expressions (arithmetic and harmonic averages) is negligible. The range of estimated $b_{h}{ }^{3}$ overlaps the range of error in the experimentally measured $b_{h}{ }^{3}$ shown in Table 2 . We note that the cube of the mean aperture is almost identical to the simulated values for $b_{h}{ }^{3}$, demonstrating that the large scale trends in the aperture field of the Hele-Shaw cell (discussed in section 2.1 above) have little influence on the cell's effective transmissivity.

Simulation results in the smooth-rough fracture gave estimates for $b_{h}{ }^{3}$ that ranged from 1.498 $1.608 \times 10^{-06} \mathrm{~cm}^{3}$. The harmonic average link transmissivity (7b) predicts the smallest $b_{h}^{3}$, followed in increasing order by the wedge expressions $(7 \mathrm{f}),(7 \mathrm{~g})$, and $(7 \mathrm{e})$, then (7d), (7c), and (7a). Predictions of $b_{h}^{3}$ for the rough-rough fracture ranged from $0.971-1.042 \times 10^{-05} \mathrm{~cm}^{3}$ in the following increasing order: (7b), (7h), (7e), (7d), (7c), (7a). Both sets of results are consistent with the behavior noted in Figures 7 and 8 . Discrepancies from experimental measurements were $22-34 \%$ for the rough-rough cell, and $32-47 \%$ for the smooth-rough cell; far outside the range defined by experimental error in the effective transmissivity measurements. We note that an increase in $\langle b>$ of $\sim 10-14 \%$ for the smooth-rough fracture and $\sim 7-10 \%$ for the rough-rough fracture would be required to account for the discrepancy between experiment and simulation; however, the error in our experimental measurement of $\langle b>(\sim 0.7 \%)$ precludes this factor as a source for the observed discrepancy. The larger range of variation for the smooth-rough fracture is consistent with the larger coefficient of variation in the aperture field. It is interesting to note that for the smooth-rough fracture, computations employing $(7 \mathrm{f})$ and $(7 \mathrm{~g})$ predicted values for $\mathrm{b}_{\mathrm{h}}{ }^{3}$ that were only marginally lower $(<2 \%)$ than predictions based on $(7 \mathrm{e})$. This observation is explained by the statistics of the measured wedge angles, which give a mean of $5.34^{\circ}$, and standard deviation of $\sim 4^{\circ}$. As a result, the ratio $(7 \mathrm{f}) /(7 \mathrm{e})$ has a mean value of 0.9917 , and is larger than 0.95 for $99 \%$ of the grid blocks in the measured aperture field; similar results are obtained for $(7 \mathrm{~g})$.

\section{Influence of spatial resolution on simulation results}

We now explore solution sensitivity to spatial resolution using aperture data from the smoothrough fracture; results for the rough-rough fracture are similar, and hence are not presented. Our original aperture data was obtained at a grid size of $0.015 \mathrm{~cm}$. We begin by replacing each set of four $(2 \times 2)$ neighboring blocks with the value in the lower left block. This "subsampling" process simulates point measurements taken at separations larger than the original grid size, such as acquired using various profilometry techniques (e.g., Reimus et al., 1993; Durham and Bonner, 
1994; Yeo et al., 1998). Recursive application of this process generated aperture fields of successively coarser resolution (up to $0.48 \mathrm{~cm}$ ). Flow was simulated on the series of aperture fields using three different formulations for the link transmissivity: the harmonic average (7b), mid-point (7c), and Stokes wedge (7f). Results (Figure 9a) show that differences between alternative estimates of $b_{h}{ }^{3}$ grow with grid size. The subsampling scheme destroys the inherent spatial correlation structure of the original fracture, thus increasing the probability of having two highly disparate aperture values adjacent to each.other. In such cases, (7b) is dominated by the smaller aperture value, leading to lower estimates of $b_{h}^{3}$ for increased grid spacing. Conversely, the larger aperture dominates in (7c), leading to an increase in estimated $b_{h}^{3}$ with increasing grid size. It is interesting to note that the $b_{h}{ }^{3}$ values estimated using ( $7 f$ ) show relatively less change with grid size. This observation may be explained as follows: even if widely disparate aperture values are brought close together by subsampling, the change in wedge angle may not be large, because the length of the "wedge" doubles at each level of coarsening.

Because the light transmission technique measures average aperture over each individual grid block, we next consider the effects of reduced spatial resolution by replacing each set of four ( 2 x2) neighboring blocks with their average aperture. Again, recursive application generates a series of aperture fields with successively coarser resolution. As the aperture field is coarsened by averaging, the aperture variance decreases progressively ( 2 orders of magnitude over 5 recursions). This contrasts significantly with the subsampling approach, in which variance remains constant through the coarsening process. Results of flow simulations (Figure 9b) show that estimates of $b_{h}^{3}$ using different link transmissivities first diverge with decreasing spatial resolution, then converge and approach $\langle b\rangle^{3}$ at the coarse end of the grid sizes considered $(0.48 \mathrm{~cm})$. The maximum difference between the different estimates of $b_{h}{ }^{3}$ occurs when the grid size is close to the correlation length. At very fine resolutions and at very coarse resolutions obtained through averaging, point-to-point variance between adjacent apertures is expected to be small; therefore, differences between the various formulations for link transmissivity are also expected to be small (see Figure 8). Results suggest that estimates of $b_{h}{ }^{3}$ are much less sensitive to spatial resolution when aperture data is collected through an averaging technique (e.g., light transmission) rather than a point sampling technique (e.g., profilometry). A point sampling technique would also result in a wider variation in the $b_{h}{ }^{3}$ values predicted using different link transmissivity expressions.

Noting that estimates for $b_{h}{ }^{3}$ using $(7 a-h)$ do not fully converge at the scale of our data resolution $(\sim 0.015 \mathrm{~cm})$, we finish this investigation by exploring the question of "How much resolution is sufficient?". High-resolution data (grid spacing $0.002 \mathrm{~cm}$ ) was obtained over a $2 \times 2 \mathrm{~cm}$ subset of the smooth-rough fracture by moving the CCD camera closer to the fracture. The normalized aperture field was then scaled to have the same mean aperture as the full fracture. As described above, data was reduced in resolution by recursively replacing each set of four (2.x2) neighboring blocks with their average aperture until a maximum grid spacing of $0.032 \mathrm{~cm}$ was obtained. Estimates of $b_{h}{ }^{3}$ obtained using different link transmissivities (Figure 9b) converge at very high resolution. A perture variation between adjacent grid blocks becomes sufficiently small that there is little difference among the various link transmissivities (see Figure 8). With the Stokes wedge and harmonic average link transmissivities, the change in $b_{h}{ }^{3}$ over the range of grid spacings between 0.015 and $0.002 \mathrm{~cm}$ is less than $2 \%$. The transmissivity estimates obtained from the highresolution field and the full field are almost identical (except for the harmonic average case at a pixel size of about $0.032 \mathrm{~cm}$ ). Based on Figure $9 \mathrm{~b}$, the resolution of $0.015 \mathrm{~cm}$ (about one-fifth of the correlation length) used in the detailed simulations presented in Section 3 appears justifiable. 


\section{Summary and implications}

Validity of the Reynolds equation for simulating saturated fracture flow was considered in a HeleShaw cell and two rough-walled analog fractures specifically designed to be representative of isotropic, spatially correlated random fields much larger than the spatial correlation length. One rough-walled analog (rough-rough) was constructed by holding two plates of textured glass in close contact; the second (smooth-rough) was fabricated from one flat and one textured plate. Transmissivity (expressed as the cube of hydraulic aperture, $b_{h}{ }^{3}$ ) was measured in all three fractures for Reynolds numbers $(R)$ ranging from somewhat below, to somewhat above one. With the exception of the largest Reynolds number considered in the rough-rough fracture $\left(R_{c}=6.8\right)$, all experimental results displayed a linear relationship between flow and gradient. This observation is consistent with the suggestion by Zimmerman and Bodvarsson [1996] that $R_{e}=1$ provides a conservative upper bound on the assumption of negligible inertial effects for flow in a roughwalled fracture.

The light transmission technique of Detwiler et al. [in press] was used to obtain high resolution aperture field data $(\sim 0.015 \mathrm{~cm})$ at the time of the hydraulic measurements with known, well defined, error. Experimental measurements of $b_{h}{ }^{3}$ were compared to the results of simulated flow on the experimentally measured aperture fields. In the Hele-Shaw cell, numerical simulations yielded a predicted value of $b_{h}{ }^{3}$ that was within experimental error of the transmissivity measurement. In the rough-walled fractures, simulated $b_{h}{ }^{3}$ exceeded the experimental values by 22$34 \%$ for the rough-rough cell, and $32-47 \%$ for the smooth-rough cell. The ranges in these estimates reflect potential errors in the aperture measurement $(\sim 1 \%$ of the mean) and alternative formulations for the link transmissivity. These predictions are far outside the range of experimental error $(\sim 1-2 \%)$. We thus conclude that in the rough-walled fractures, most of the discrepancy between the predicted and experimental estimates of $b_{h}{ }^{3}$ is due to the inaccuracy of the Reynolds equation in describing the physics of the flow process.

We explored several alternative formulations for the link transmissivity in the finite-difference discretization of the Reynolds equation. Simple averaging schemes and formulations based on approximations to Stokes flow were considered. Use of the simple harmonic average link transmissivity yielded the lowest overall effective transmissivity for simulations in our measured aperture fields. It is also interesting to note that attempts to correct the Reynolds equation with local Stokes flow and other approximations are not very effective in refining the predictions of $b_{h}{ }^{3}$. Even (7f), which is based on an exact solution to the Stokes equations in the precisely known local geometry of the smooth-rough fracture, modifies the $b_{h}{ }^{3}$ estimated using (7e) by only about $2 \%$. The implication is that three-dimensional Stokes flow effects have non-local manifestations that are difficult to describe with local corrections.

We also investigated the sensitivity of the simulated effective transmissivity to spatial resolution of the aperture measurements. The full-field aperture measurements were-coarsened using two schemes, one simulating larger averaging areas and another simulating increased spacing between point measurements. The simulated effective transmissivity was found to be more sensitive to resolution in the case of point measurements. Furthermore, when the point measurements were used, alternative formulations of the link transmissivity resulted in a wider range of simulated effective transmissivities. In general, the different link transmissivity formulations yield widely different estimates of the overall effective transmissivity when the resolution is much coarser than the aperture correlation length. Our investigation of the influence of spatial resolution of aperture field data on estimates of $b_{h}^{3}$ demonstrates that area averaged measurements obtained at a spacing of about $1 / 5$ th of a correlation scale lead to sufficiently convergent predictions of fluid flow (within 2\%). 
To our knowledge, this is the first detailed evaluation of the Reynolds equation on extensive, highly accurate, measured aperture fields. Zimmerman and Bodvarsson [1996] suggest that the ratio of mean aperture to twice the characteristic scale of variability should be much smaller than 1 for the Reynolds equation to be valid. Although this ratio is roughly an order of magnitude smaller than 1 for the experimental fractures $(0.14$ and 0.078 for the rough-rough and smooth-rough, respectively), it is apparently not sufficiently small for the Reynolds equation to be valid. It is interesting to note that there is less discrepancy between the experimental and predicted fracture transmissivity for the rough-rough fracture, even though the mean aperture to spatial correlation scale is larger. However; the coefficient of variation of the aperture field, which may be viewed as another measure of deviation from the parallel plate geometry, is smaller for the rough-rough fracture.

While our analog rough-walled fractures were designed to provide a straightforward test for the Reynolds Equation in an isotropic, spatially correlated field, the characteristics of the aperture field (mean, variance, spatial correlation length) fall within the large range of that which has been measured in natural fractures to date. The mean aperture values of 0.0124 and $0.0225 \mathrm{~cm}$ for our rough-walled fractures are in the lower end of the range covered in measurements of natural fractures by Gale [1990] and Hakami [1989], as reported by Zimmerman and Bodvarsson [1990]. The standard deviations of 0.0043 and $0.0062 \mathrm{~cm}$ for our fractures are also in the low end of the range covered in the aforementioned data. However, Brown [1995] characterized the spatial structure of the aperture field in several natural fracture traces (lengths ranging from 13 to $52 \mathrm{~mm}$ ) and found standard deviations of the aperture in the range of $0.0017-0.018 \mathrm{~cm}$, which are smaller than the corresponding values for our analog fractures. He also estimated "mismatch length scales" (corresponding roughly to about one-fifth of the spatial correlation length) in the range of 0.013 $0.3 \mathrm{~cm}$. The corresponding range of about $0.065-1.5 \mathrm{~cm}$ for the correlation length, encompasses the value of $0.08 \mathrm{~cm}$ associated with our analog fracture.

Natural fractures of ten have the additional complication of long-range undulations with characteristic scales of a few $\mathrm{cm}$ superimposed on small-scale roughness [e.g., Tsang and Witherspoon, 1983; Power and Tullis, 1991; Brown, 1995]. These undulations are primarily due to the movement of one rough surface relative to the other. Above the scale of the undulation, Brown [1995] notes that the aperture field, which is the composite topography obtained from the two rough surfaces, typically does not exhibit large-scale structures and is statistically stationary. Work by Thompson and Brown [1991] employing the Reynolds equation showed that such longrange undulations act to channelize flow and that the influence of these features on both effective transmissivity and the degree of channelization increases as the separation distance between two rough fracture surfaces is decreased. Because the length scale for these features are large with respect to those of the small-scale roughness, it is likely that while such studies will suffer from inaccuracies of the Reynolds equation as shown in this paper, the trends seen in transmissivity as a function of separation between the fracture surfaces will remain.

How well we need to model the flow through a fracture is, of course, a function of the question being considered. As has been shown in many contexts, and at a large number of scales, accurate representation of the flow field is critical in the modeling and understanding of more complex fundamental processes such as solute transport, two-phase flow, and interphase mass transfer. Such will also be true in individual fractures. Future work should focus on numerical simulation of the full three-dimensional Navier-Stokes equations in fractures where the geometry of the field is systematically varied. Approaches using solution techniques based on Lattice-Boltzman methods show considerable promise for such studies [Stockman et al.,1998]. Such efforts will help to further clarify the mechanisms controlling fluid flow in rough-walled fractures and possibly motivate improved two-dimensional representations. 


\section{Acknowledgments}

This work was supported by the U.S. Department of Energy's Basic Energy Sciences Geoscience Research Program under contract numbers DE-FG03-99ER14944 (Oklahoma State University), DE-FG03-96ER14590 (University of Colorado at Boulder), and DE-AC04-94AL85000 (Sandia National Laboratories). We thank Mehdi Eliassi, Robert Holt, Yvonne Tsang (who also brought the work by $Y$ eo et al. [1998] to our attention), and an anonymous reviewer for their constructive reviews of the manuscript. We also thank Donald Fox for assistance in test cell assembly. Experiments were conducted at the Flow Visualization and Processes Laboratory at Sandia National Laboratories.

\section{References}

Sandia is a multiprogram laboratory operated by Sandia Corporation, a Lockheed Martin Company, for the United States Deparment of Energy under contract DE-AC04-94AL85000.

Bakr, A.A., L.W. Gelhar, A.L. Gutjahr, and J.R. MacMillan, Stochastic analyses of spatial variability in subsurface flows, 1 . comparison of one- and three-dimensional flows, Water Resources Research, 14(2), 263-272, 1978.

Bear, J., Hydraulics of Groundwater, McGraw-Hill Inc., New York, 569 pp., 1979.

Bear, J., Modeling flow and contaminant transport in fractured rocks, in Flow and contaminant transport in fractured rock, Bear, J., C.f. Tsang, and G. de Marsily eds., Academic Press, San Diego, 560 pp., 1993.

Bird, R.B., W.E. Stewart, and E.N. Lightfoot, Transport Phenomena, John. Wiley and Sons, NY, 780 pp., 1960.

Brown, S.R., Fluid flow through rock joints: The effect of surface roughness, J. Geophysical Research, 92(B2), 1337-1347, 1987.

Brown, S.R., Transport of fluid and electric current through a single fracture, J._Geophysical Research, 94(B7), 9429-9438, 1989.

Brown, S.R., Simple mathematical model of a rough fracture, J. Geophysical Research, 100(B4), 5941-5952, 1995.

Brown, S.R., H.W. Stockman, and S.J. Reeves, Applicability of the Reynolds equation for modeling fluid flow between rough surfaces, Geophysical Research Letters, 22, 2537-2540, 1995.

Brown, S., A. Caprihan, and R. Hardy, Experimental observation of fluid flow channels in a single fracture, J. Geophysical Research, 103 (B3), 5125-5132, 1998.

Cordes, C. and W. Kinzelbach, Comment on: "Application of mixed hybrid finite-element approximations in a groundwater flow model: Luxury or necessity?", by R. Mose, P. Siegel, P. Ackerer, and G. Chavent, Water Resources Research, 32(6), 1905-1909, 1996.

Dagan, G., Models of groundwater flow in statistically homogeneous porous formations, Water Resources Research, 15(1), 47-63, 1979.

Dagan, G., Flow and Transport in Porous Formations, Springer Verlag, Berlin, 1989.

Detwiler, R. L., S. E. Pringle, and R. J. Glass, Measurement of fracture aperture-fields using transmitted light: An evaluation of measurement errors and their influence on simulations of flow and transport through a single fracture, Water Resources Research, in press. 
Durham, W.B., and B.P. Bonner, Self-propping and fluid flow in slightly offset joints at high effective pressures, J. Geophysical Research, 99(B5), 9391-9399, May 10, 1994 :

Gale, J., R. MacLeod, and P. LeMessurier, Site characterization and validation - Measurement of flow rate, solute velocities and aperture variation in natural fractures as a function of normal and shear stress, stage 3, Stripa Project Report 90-11, Swedish Nuclear Fuel and Waste Management Co., Stockholm, 1990.

Ge, S., A governing equation for fluid flow in rough fractures, Water Resources Research, 33(1), 53-61, 1997.

Gelhar, L.W., Applications of stochastic models to solute transport in fractured rocks, Swedish Nuclear Fuel and Waste management Company, SKB Tech. Report 87-05, Stockholm, Sweden, 1987.

Gelhar, L.W., Stochastic Subsurface Hydrology, Prentice-Hall, Englewood Cliffs, New Jersey 1993.

Glass, R.J., M.J. Nicholl, and M.E. Thompson, Comparison of measured and calculated permeability for a saturated, rough-walled fracture, Presented at the Fall 1991 Meeting of the Am. Geophys. Union, (H51D-11) EOS, 72 (216), 1991.

Glass, R.J., Modeling gravity-driven fingering in rough-walled fractures using modified percolation theory, Proceedings of the Fourth Annual International Conference on High Level Radioactive Waste Management, 2042-2052, American Nuclear Society, Las Vegas, Nevada, April 26-30, 1993.

Glass, R.J. and M.J. Nicholl, Quantitative visualization of entrapped phase dissolution within a horizontal flowing fracture, Geophysical Research Letters, 22(11), 1413-16, 1995.

Glass, R.J., M.J. Nicholl, and L. Yarrington, Development and experimental evaluation of models for low capillary number two-phase flows in rough walled fractures relevant to natural gradient conditions, Sandia National Laboratories, Albuquerque, NM, SAND96-2820, 116 pp., 1997.

Glass, R.J., M.J. Nicholl, and L. Yarrington, A modified invasion percolation model for low capillary number immiscible displacements in horizontal rough walled fractures: Influence of local in-plane curvature, Water Resources Research, 34(12), 3215-3234, 1998.

Hakami, E., Water flow in single rock joints, SKB Technical Report 89-08, Swedish Nuclear Fuel and Waste Management Co., 1989.

Hakami, E., and E. Larsson, Aperture measurements and flow experiments on a single natural fracture, Int. J. Rock Mechanics Mineral Science and Geomechanics Abstracts, 33, (4), 395-404, 1996.

Landau, L.D., and Lifshitz, Electrodynamics of continuous media, Pergamon Press, New York, 417 pp., 1960.

Langlois, W.E., Slow Viscous Flow, The MacMillan Company, New York, 1964.

Larabi, A., and F. De Smedt, Solving three-dimensional hexahedral finite element groundwater models by preconditioned conjugate gradient methods, Water Resources Research, 30(2), 509$521,1994$. 
Longwell, P.A., Mechanics of fluid flow, McGraw-Hill, New York, 433 pp., 1966.

Nicholl, M.J., R.J. Glass, and H.A. Nguyen, Small-scale behavior of single gravity-driven fingers in an initially dry fracture, Proceedings of the Fourth Annual International Conference on High Level Radioactive Waste Management, 2023-2032, American Nuclear Society, Las Vegas, Nevada, April 26-30, 1993. (SNL paper SAND92-2790C).

Nicholl; M.J., R.J. Glass, and H.A. Nguyen, Wetting front instability in an initially wet unsaturated fracture, Proceedings of the Fourth Annual International Conference on High Level Radioactive Waste Management, 2061-2070, American Nuclear Society, Las Vegas, Nevada, April 26-30, 1993 (SNL paper SAND92-2791C).

Nicholl, M.J., and R.J. Glass, Wetting phase permeability in a partially saturated horizontal fracture, Proceedings of the Fifth Annual International Conference on High Level Radioactive Waste Management, 2007-2019, American Nuclear Society, Las Vegas, Nevada, May 22-26, 1994.

Nicholl, M.J., R.J. Glass, and S.W. Wheatcraft, Gravity-Driven infiltration instability in initially dry nonhorizontal fractures, Water Resources Research, 30 (9), 2533-2546, 1994.

Patir, N. and A.S. Cheng, An average flow model for determining effects of three-dimensional roughness on partial hydrodynamic lubrication, J. Lubrication Tech., 100, 12-17, 1978.

Persoff, P., and K. Pruess, Two-phase flow visualization and relative permeability measurement in rough-walled fractures, Water Resources Research, 31 (5), 1175-86, 1995.

Piggot, A.R., and D. Elsworth, Laboratory assessment of equivalent apertures of a rock fracture, Geophysical Research Letters, 20 (13), 1387-90, 1993.

Power, W.L., and T.E. Tullis, Euclidean and fractal models for the description of rock surface roughness, J. of Geophysical Research, 96(B1), 415-424, January 10, 1991.

Reimus, P., R. Glass, and B. Robinson, Aperture characteristics, saturated fluid flow, and tracer transport calculations for a natural fracture, Proceedings of the Fourth Annual International Conference on High Level Radioactive Waste Management, 2009-2016, American Nuclear Society, Las Vegas, Nevada, April 26-30, 1993.

Reynolds, O., On the theory of lubrication, Phil. Trans. Roy. Soc. London, 177, 157 - 234, 1886.

Stockman, H.A., R.J. Glass, C.A. Cooper, and H. Rajaram, Accuracy and computational efficiency in 3D dispersion via Lattice Boltzmann: Models for dispersion in rough fractures and double-diffusive fingering, International Journal of Modern Physics C, 9(8), 1-13, 1998.

Thompson, M.E., and S.R. Brown, The effect of anisotropic surface roughness on flow and transport in fractures, J. of Geophysical Research, 96(B13), 21, 923-21, 932, December 10, 1991 .

Tsang, Y.W., and P.A. Witherspoon, Hydromechanical behavior of a deformable rock fracture, J. Geophysical Research, 86(B10), 9287-98, 1981.

Tsang, Y.W., and P.A. Witherspoon, The dependence of fracture mechanical and fluid properties on fracture roughness and sample size, J. Geophysical Research, 88(B3), 2359-2366, 1983. 
Tsang, Y.W. and C.F. Tsang, Flow channeling in a single fracture as a two-dimensional strongly heterogeneous permeable medium, Water Resources Research 25(9), 2076-2080, 1989.

Waite, M.E., S. Ge, H. Spetzler, and D.B. Bahr, The effect of surface geometry on fracture permeability; A case study using a sinusoidal fracture, Geophysical Research Letters, 25 (6), 813816, 1998.

West, W., Absorption of electromagnetic radiation, in the Optics source book, S.P. Parker ed., McGraw-Hill, New York, 399 pp., 1988.

Yeo, I.W., M.H. De Freitas, and R.W. Zimmerman, Effect of shear displacement on the aperture and permeability of a rock fracture, Int. J. Rock Mechanics Mineral Science, 35(8), 1051-1070, Pergamon Press, 1998.

Zimmerman, R.W., S. Kumar, and G.S. Bodvarsson, Lubrication theory analysis of the permeability of rough-walled fractures, Int. J. Rock Mechanics Mineral Science and Geomechanics Abstracts, 28(4), 325-331, Pergamon Press, 1991.

Zimmerman, R.W., and G.S. Bodvarsson, Hydraulic conductivity of rock fractures, Transport in Porous Media, 23, 1-30, 1996. 


\section{List of Figures}

Figure 1: Fracture test tell: Transparent analog fractures are fixed within a test cell that allows imposition of boundary conditions, application of normal pressure to the fracture plane (roughwalled fractures only), and transmission of light right up to the fracture boundaries. a) Plan view of test cell. Also shows the stepped neutral density wedge used as a reference to adjust images for small oscillations in light source intensity. b) Cross-section $X-X$ ' for rough-walled fractures. $c$ ) Cross-section X-X' for Hele-Shaw cell. After Detwiler et al.[in press].

Figure 2: Aperture distributions: Aperture distributions are shown for the three analog fractures; percent occupancy is shown as a function of measured aperture. Note that values for the two rough-walled fractures have been multiplied by a factor of 10 .

Figure 3: Rough-walled aperture fields: Arbitrarily chosen subsets $(3 \times 3 \mathrm{~cm})$ of the roughwalled aperture fields show the basic aperture structure produced by the textured glass plates; pattern of the glass is clearly seen in the image of the smooth-rough fracture. These sets are representative of aperture over the whole fields.

Figure 4: Aperture variograms: Point-to-point variance along the primary axes of each fracture are shown as a function of separation distance on $\log -\log$ axes. Data along the diagonal axes is consistent with that taken along the primary axes, and hence not shown. When normalized to system variance, data for the smooth-rough and rough-rough fractures are indistinguishable. Spatial correlation within the smooth-rough and rough-rough fractures is isotropic, while the HeleShaw cell exhibits an anisotropic correlation.

Figure 5: Flow data: Experimental measurement of fracture transmissivity. The left-hand-side of (3) is plotted as a function of hydraulic gradient; slope of the data gives $b_{h}{ }^{3}$.

Figure 6: Conceptual models: a) Approximation of the fracture as a series of wedges, no assumptions are made regarding symmetry of the wedges. b) Known geometry of the smoothrough fracture is used to approximate the fracture as a series of wedges with all variation in one surface. c) Conceptualization of radial flow in a region bounded by two arcs (shown in gray). For the case shown in $6 \mathrm{~b}$, only that portion of the annular region located above the center line is considered in the derivation of (7f). Both the upper and lower halves are considered in the derivation of (7h). d) Approximation of the fracture as a series of symmetric wedges.

Figure 7: Effects of wedge angle: Equations (7f-h) are corrections to (7e) that account for specific geometry. The asymmetric wedge formulations $(7 \mathrm{f}, \mathrm{g})$ impart a significantly larger correction than does that for a symmetric wedge $(7 \mathrm{~h})$.

Figure 8: Sensitivity, of link transmissivity to local aperture variability: Response of link transmissivity is shown as a function of $r$, the size ratio of adjacent apertures.for equations (7b) and (7e-h). Grid spacing was arbitrarily set equal to $b_{i, j}$ for formulations (7f-h) which are explicitly dependent on the wedge angle. Results show that for small $r$, little difference exists between the various formulations. At large $r$, where significant differences exist between the various formulations, assumptions of depth averaging used to develop the wedge flow approximations (7f-g) may become tenuous. 
Figure 9: Effects of discretization scale: Resolution of the measured aperture data was reduced by: (a) subsampling and: (b) averaging. A higher resolution data set $(0.002 \mathrm{~cm})$. was measured on a $2 \times 2 \mathrm{~cm}$ section of the fracture to consider resolutions ranging from 0.002 to 0.032 $\mathrm{cm}$. Simulation results are scaled to the cube of mean aperture $\left(\langle\mathrm{b}\rangle^{3}\right)$. Experimental results and predictions based on stochastic theory (equation (4)) are shown as horizontal lines. Results show that choosing grid spacing similar to the correlation scale will make numerical simulations particularly sensitive to choice of link transmissivity. 


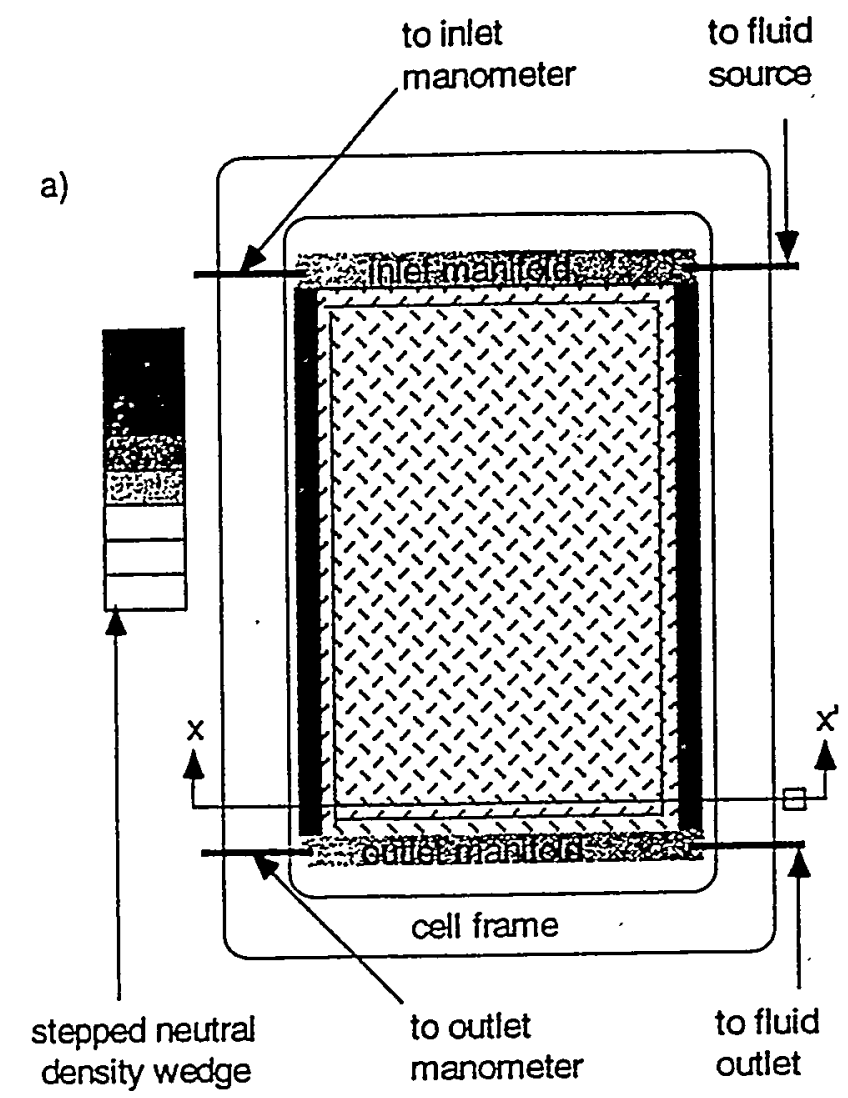

b)

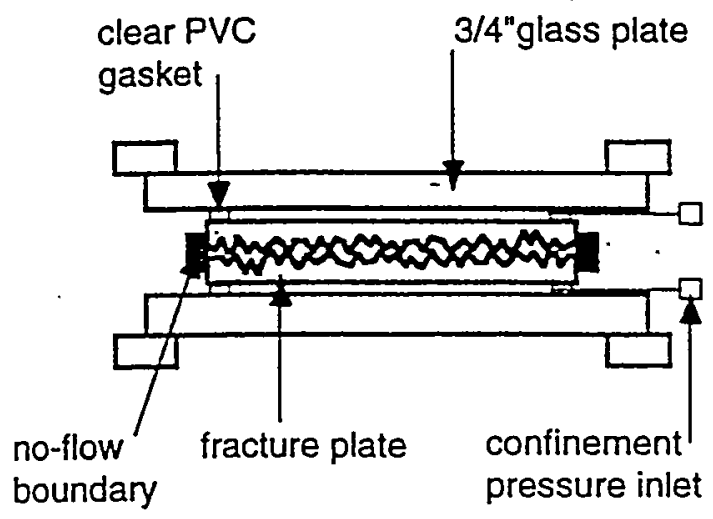

c)

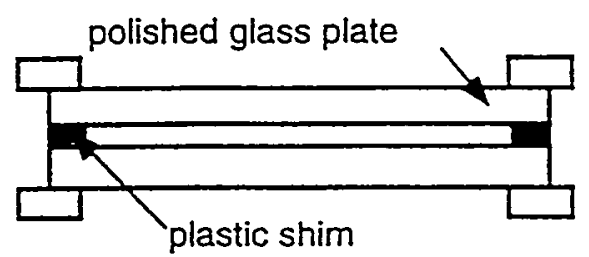

Figure 1 


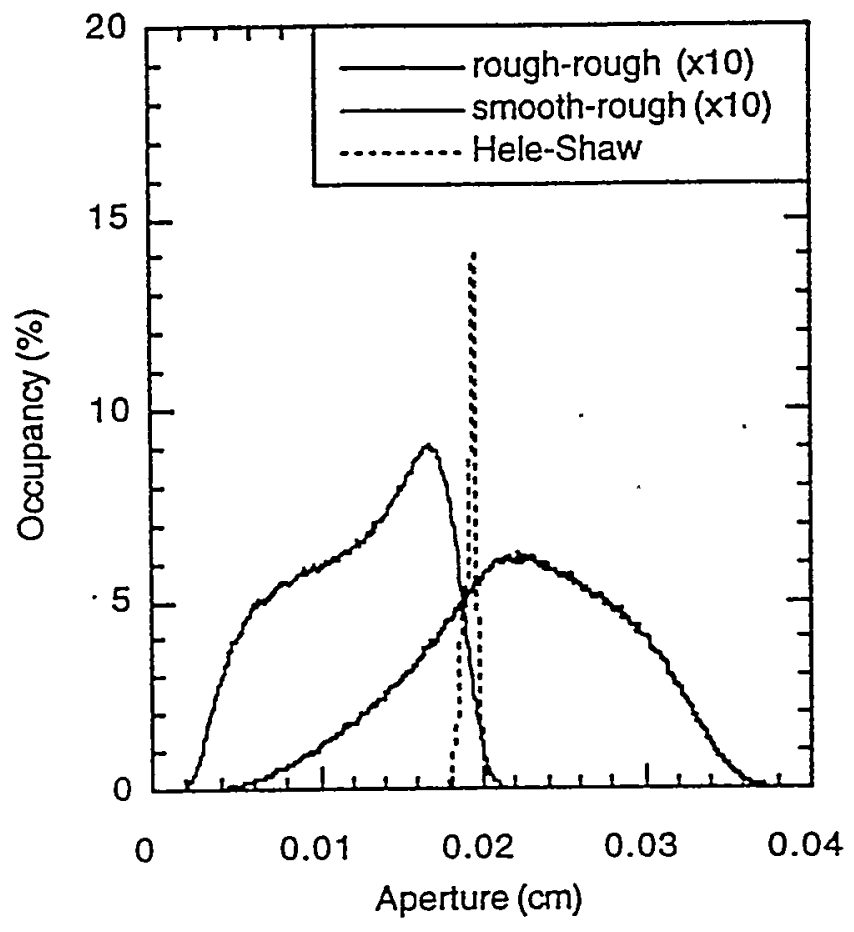

- Figure 2 


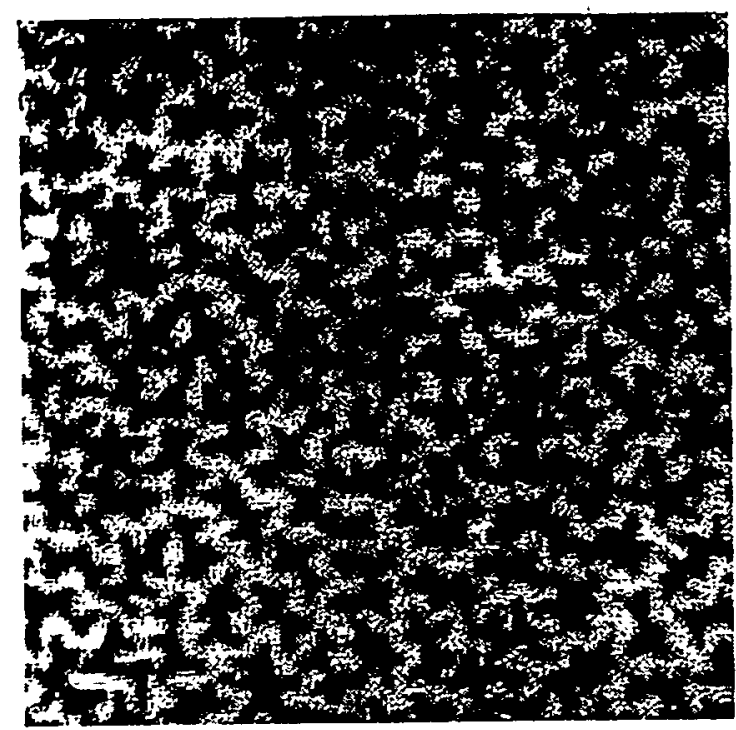

smooth-rough

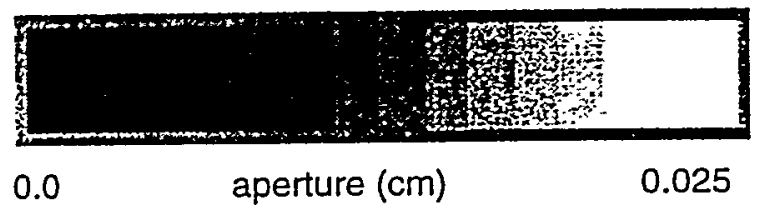

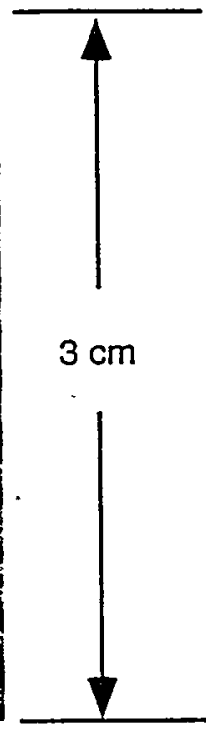

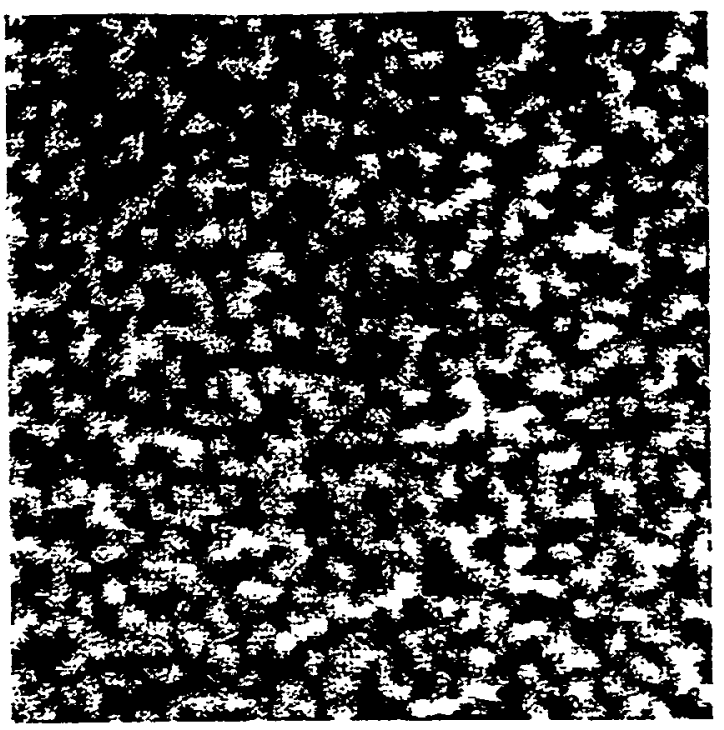

rough-rough

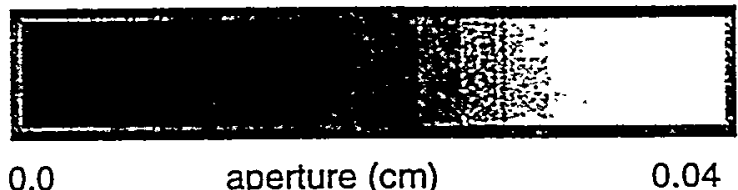

0.04

Figure 3 


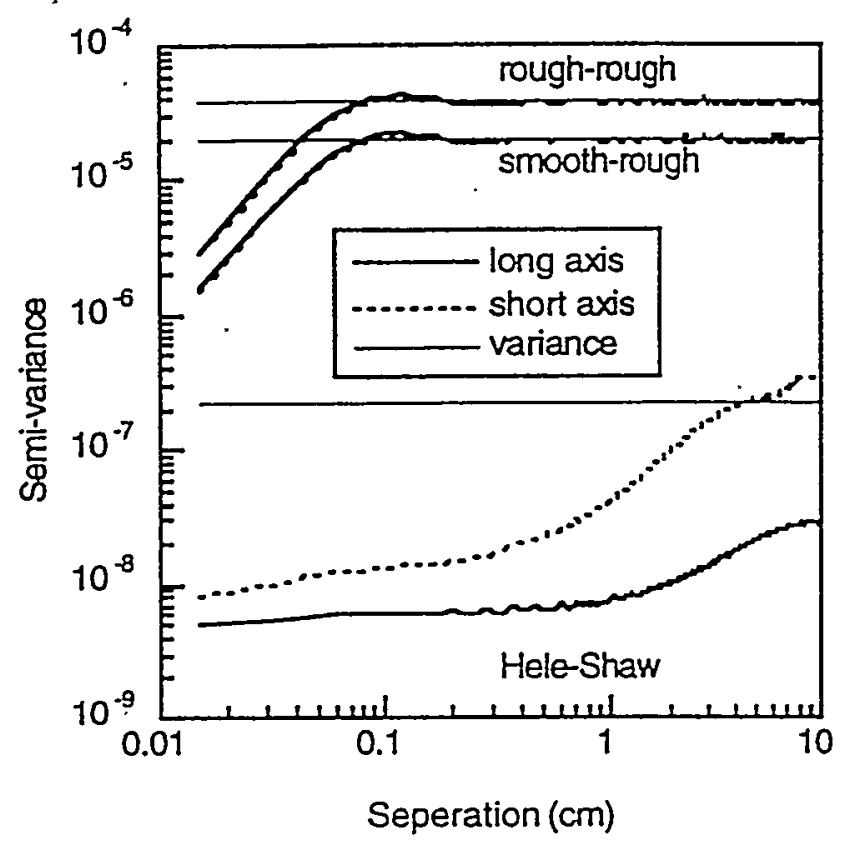

Figure 4 


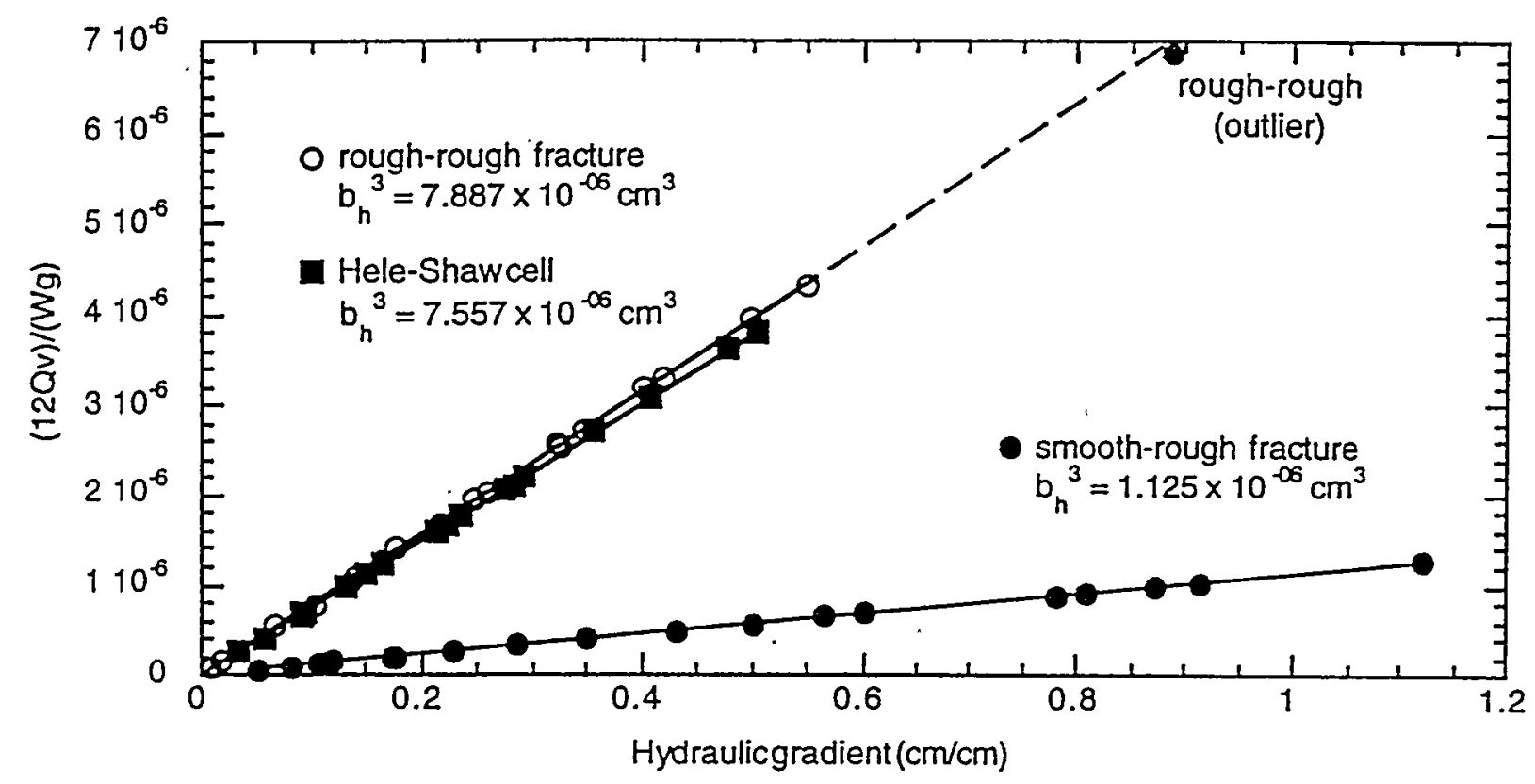

- Figure 5 
a)

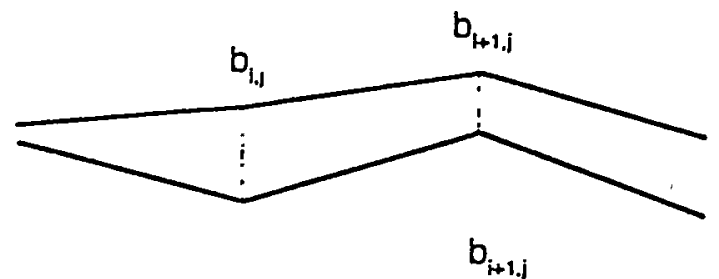

b)

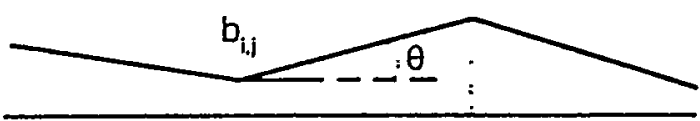

c)

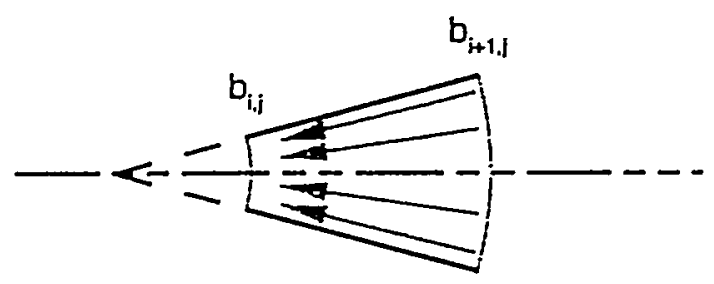

d)

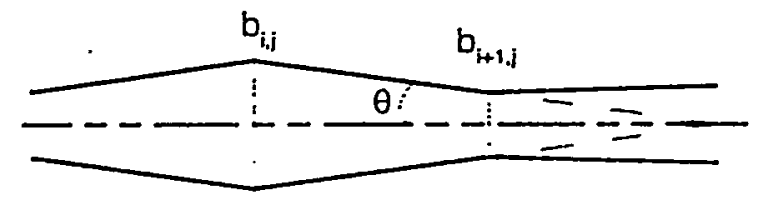

Figure 6 


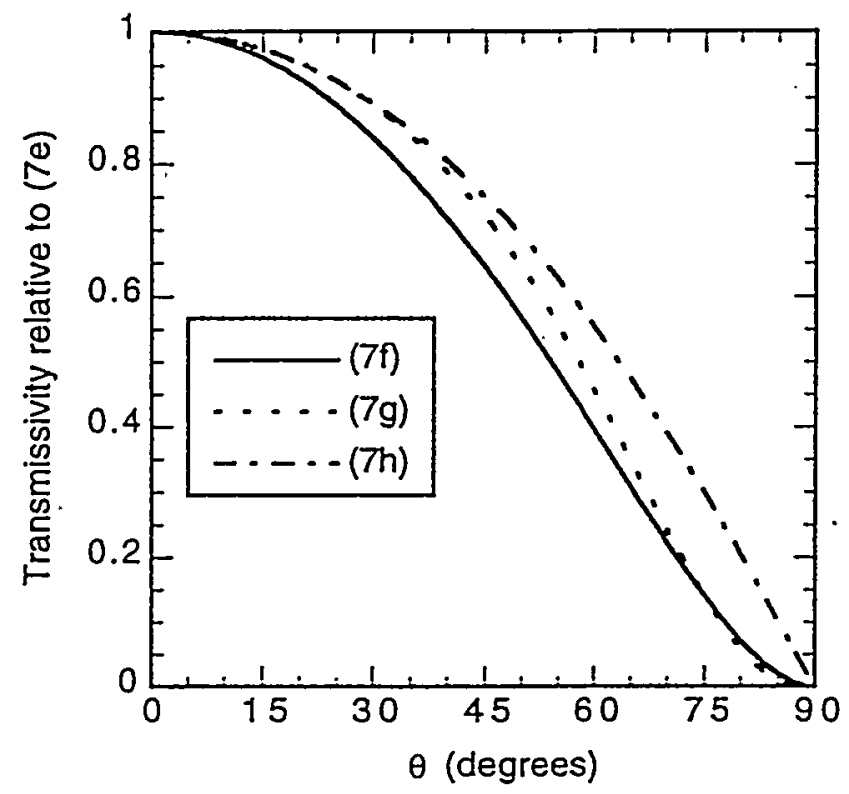

Figure 7 


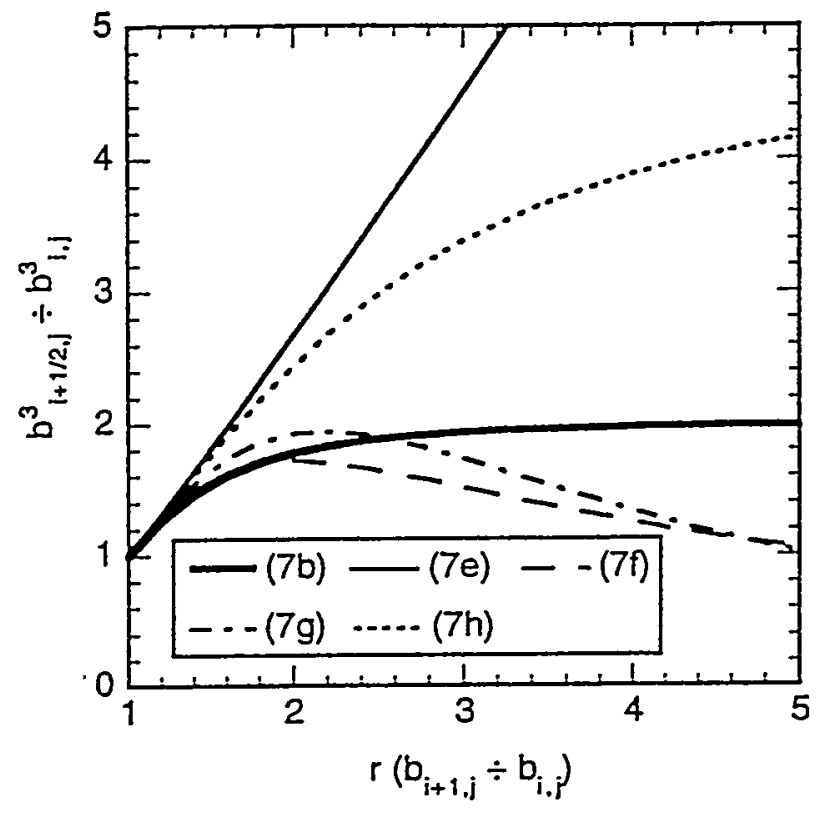

Figure 8 

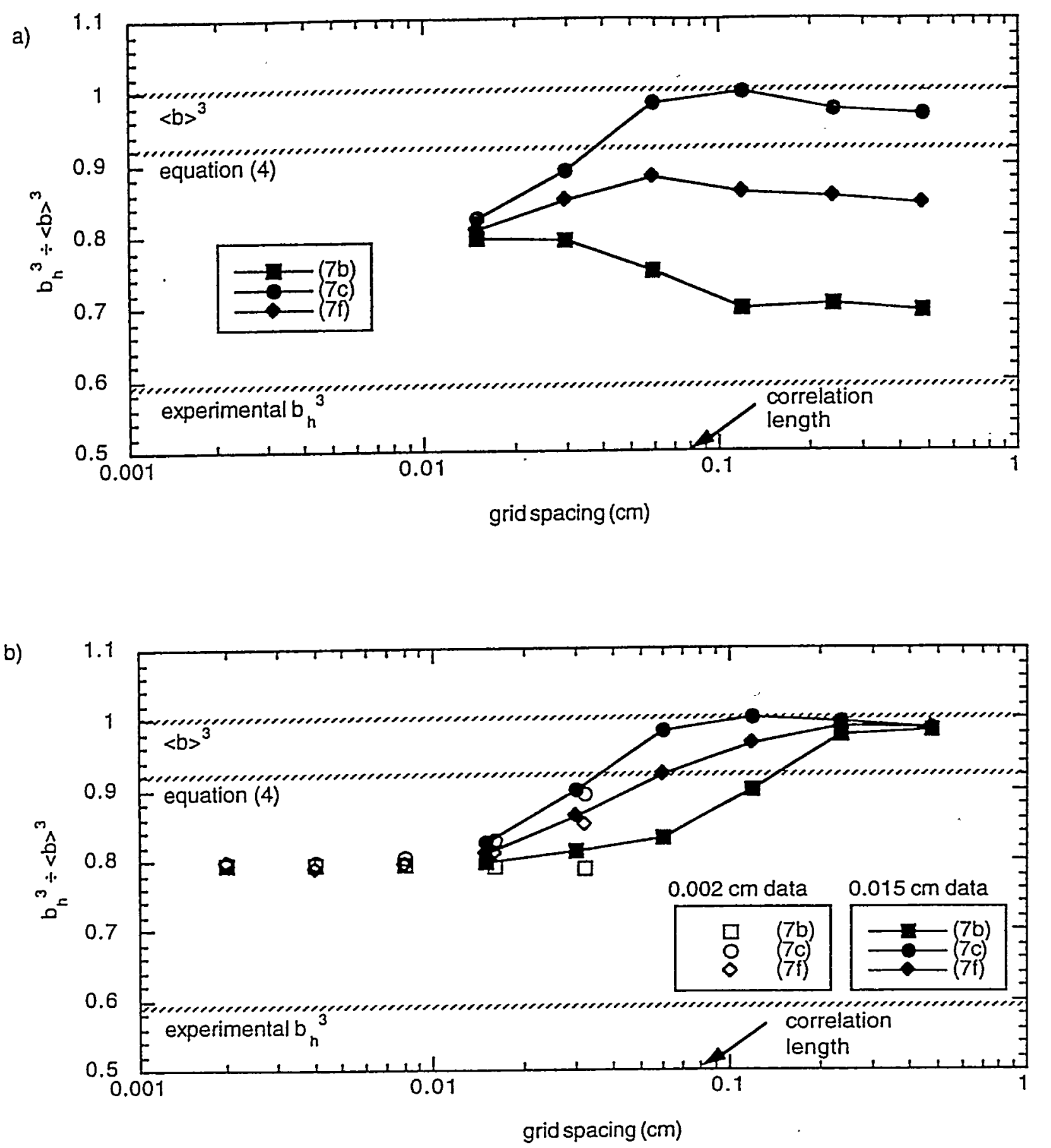

\footnotetext{
- Figure 9
} 
Table 1: Measured aperture statistics

\begin{tabular}{|l|l|l|l|}
\hline Distribution Statistic & Hele-Shaw & rough-rough & smooth-rough \\
\hline Minimum & $1.127 \times 10^{-02} \mathrm{~cm}$ & $3.000 \times 10^{-03} \mathrm{~cm}$ & $1.412 \times 10^{-03} \mathrm{~cm}$ \\
\hline Maximum & $3.011 \times 10^{-02} \mathrm{~cm}$ & $4.040 \times 10^{-02} \mathrm{~cm}$ & $2.498 \times 10^{-02} \mathrm{~cm}$ \\
\hline Mean $(<b>)$ & $1.942 \times 10^{-02} \mathrm{~cm}$ & $2.255 \times 10^{-02} \mathrm{~cm}$ & $1.240 \times 10^{-02} \mathrm{~cm}$ \\
\hline Standard deviation $(\sigma)$ & $4.634 \times 10^{-04} \mathrm{~cm}$ & $6.183 \times 10^{-03} \mathrm{~cm}$ & $4.428 \times 10^{-03} \mathrm{~cm}$ \\
\hline $\begin{array}{l}\text { Coefficient of variation } \\
(\sigma /<b>)\end{array}$ & $2.387 \times 10^{-02}$ & $2.742 \times 10^{-01}$ & $3.572 \times 10^{-01}$ \\
\hline Total RMS error ${ }^{(1)}$ & $0.65 \%$ & $0.68 \%$ & $0.70 \%$ \\
\hline $\begin{array}{l}\text { Correlation length }(\lambda)- \\
\text { long axis }\end{array}$ & & $0.08 \mathrm{~cm}$ & $0.08 \mathrm{~cm}$ \\
\hline $\begin{array}{l}\text { Correlation length }(\lambda)- \\
\text { short axis }\end{array}$ & & $0.08 \mathrm{~cm}$ & $0.08 \mathrm{~cm}$ \\
\hline
\end{tabular}

(1) with respect to mean aperture 
Table 2: Experimental flow data

\begin{tabular}{|l|c|c|l|l|c|}
\hline & $\begin{array}{c}\text { Estimated } \\
\mathbf{b}_{\mathrm{b}}{ }^{3}\left(\mathbf{c m}^{3}\right)\end{array}$ & $\mathbf{R}^{2}$ & $\begin{array}{l}68 \% \text { confidence } \\
\text { interval }\left(\mathbf{c m}^{3}\right)^{(1)}\end{array}$ & $\begin{array}{l}\text { Bounds on } \\
\mathbf{b}_{\mathrm{h}}{ }^{3}\left(\mathbf{c m}^{3}\right)^{(2)}\end{array}$ & $\mathbf{R}_{\mathrm{c}}^{(3)}$ \\
\hline Hele-Shaw & $7.557 \times 10^{-06}$ & 0.9997 & $\begin{array}{l}7.541- \\
7.573 \times 10^{-06}\end{array}$ & $\begin{array}{l}7.443- \\
7.611 \times 10^{-06}\end{array}$ & $0.25-3.6$ \\
\hline rough-rough & $7.887 \times 10^{-06}$ & 0.9998 & $\begin{array}{l}7.874- \\
7.899 \times 10^{-06}\end{array}$ & $\begin{array}{l}7.770- \\
7.939 \times 10^{-06}\end{array}$ & $0.063-4.3$ \\
\hline smooth-rough & $1.125 \times 10^{-06}$ & 0.9999 & $\begin{array}{l}1.125- \\
1.126 \times 10^{-06}\end{array}$ & $\begin{array}{l}1.118- \\
1.132 \times 10^{-06}\end{array}$ & $0.055-1.3$ \\
\hline
\end{tabular}

(1) $68 \%$ confidence interval for $b_{b}{ }^{3}$ was obtained from the regression statistics

(2) Includes potential bias error associated with fluid viscosity and underestimation of differential head.

${ }^{(3)} R_{e}$ was estimated using mean aperture as a characteristic length scale 
Table 3: Limiting behavior of nondimensional link transmissivity $\left(b^{3}{ }_{1+1 / 2 \sqrt{ }} \div b^{3}{ }_{1 \sqrt{ }}\right)$ as $r \rightarrow \infty$

\begin{tabular}{|l|c|}
\hline Expression for link transmissivity & behavior as $\mathbf{r} \rightarrow \infty$ \\
\hline arithmetic average (7a) & $r^{3 / 2}$ \\
\hline harmonic average (7b) & 2 \\
\hline mid-point (7c) & $r^{3 / 8}$ \\
\hline geometric (7d) & $r^{3 / 2}$ \\
\hline Reynolds wedge (7e) & $2 r$ \\
\hline Stokes wedge (7f) & 0 \\
\hline modified Reynolds wedge (7g) & 0 \\
\hline symmetric wedge (7h) & $3 b_{i, j} \div(2 \Delta x)$ \\
\hline
\end{tabular}


Table 4: Simulation results

\begin{tabular}{|l|c|c|c|}
\hline $\begin{array}{l}\text { Link transmissivity } \\
\text { expression }\end{array}$ & $\begin{array}{c}\text { Hele-Shaw cell } \\
\left(\mathbf{c m}^{3}\right)\end{array}$ & $\begin{array}{c}\text { Smooth-Rough } \\
\text { cell }\left(\mathbf{c m}^{3}\right)\end{array}$ & $\begin{array}{c}\text { Rough-Rough } \\
\text { cell }\left(\mathbf{c m}^{3}\right)\end{array}$ \\
\hline arithmetic (7a) & $\begin{array}{c}7.326 \times 10^{-06} \\
(7.185-7.470)^{(1)}\end{array}$ & $\begin{array}{c}1.608 \times 10^{-06} \\
(1.575-1.642)^{(1)}\end{array}$ & $\begin{array}{c}1.021 \times 10^{-05} \\
(1.000-1.042)^{(1)}\end{array}$ \\
\hline harmonic (7b) & $\begin{array}{c}7.324 \times 10^{-06} \\
(7.182-7.468)^{(1)}\end{array}$ & $\begin{array}{c}1.530 \times 10^{-06} \\
(1.498-1.562)^{(1)}\end{array}$ & $\begin{array}{c}0.991 \times 10^{-05} \\
(0.971-1.011)^{(1)}\end{array}$ \\
\hline geometric (7c) & & $\begin{array}{c}1.568 \times 10^{-06} \\
(1.536-1.601)^{(1)}\end{array}$ & $\begin{array}{c}1.006 \times 10^{-05} \\
(0.986-1.026)^{(1)}\end{array}$ \\
\hline midpoint (7d) & & $\begin{array}{c}1.582 \times 10^{-06} \\
(1.550-1.616)^{(1)}\end{array}$ & $\begin{array}{c}1.011 \times 10^{-05} \\
(0.990-1.032)^{(1)}\end{array}$ \\
\hline Reynolds wedge (7e) & & $\begin{array}{c}1.582 \times 10^{-06} \\
(1.550-1.616)^{(1)}\end{array}$ & $\begin{array}{c}1.005 \times 10^{-05} \\
(0.985-1.025)^{(1)}\end{array}$ \\
\hline Stokes wedge (7f) & & $\begin{array}{c}1.554 \times 10^{-06} \\
(1.522-1.587)^{(1)}\end{array}$ & \\
\hline modified Reynolds wedge (7g) & & $\begin{array}{c}1.558 \times 10^{-06} \\
(1.526-1.591)^{(1)}\end{array}$ & \\
\hline symmetric wedge (7f) & & & $0.994 \times 10^{-05}$ \\
& & & \\
\hline
\end{tabular}

(1) range of possible values rising from error in the measured mean aperture 